\title{
Alkyl and Aryl Thiol Addition to [1.1.1]Propellane : Scope and Limitations of a Fast Conjugation Reaction
}

\section{Baer, Robin M.}

2018-01-26

Baer, R M , Kirschner , S , Nieger, M \& Bräse , S 2018 , ' Alkyl and Aryl Thiol Addition to [1.1.1]Propellane : Scope and Limitations of a Fast Conjugation Reaction ' , Chemistry: A European Journal , vol. 24 , no. 6 , pp. 1373-1382 . https://doi.org/10.1002/chem.201704105

http://hdl.handle.net/10138/324815

https://doi.org/10.1002/chem.201704105

unspecified

acceptedVersion

Downloaded from Helda, University of Helsinki institutional repository.

This is an electronic reprint of the original article.

This reprint may differ from the original in pagination and typographic detail.

Please cite the original version. 


\title{
Alkyl and aryl thiol addition to [1.1.1]propellane - scope and limitations of a fast conjugation reaction
}

\author{
Robin M. Bär, ${ }^{[a]}$ Stefan Kirschner, ${ }^{[a]}$ Martin Nieger, ${ }^{[b]}$ Stefan Bräse ${ }^{*},{ }^{[a],[c]}$
}

\begin{abstract}
Herein we report the addition of different thiols to the strained carbon-carbon bond of [1.1.1]propellane (1). We investigated the reaction pathway, performed the addition with substituted thiols, hydrogen sulfide and protected cysteine and verified further modifications of the products. The clean reaction proceeds probably through a radical chain process as we confirmed with different deuterium labelling experiments. It shows great functional group tolerance as halogen-, hydroxy-, methoxy-, carboxy-, amino- and nitro-substituted thiols could be added to 1 with few by-products in $16-90 \%$ yield. Oxidation of the products offers a tuning of the polarity and subsequent reactions of the products. The "click"-type reaction proceeds even faster with selenols as we show in a proof-of-concept. The thiol addition to $\mathbf{1}$ offers a facile tool for surface modifications, conjugations and tuning of hydrophilicity in bio- and medicinal chemistry compounds.
\end{abstract}

\section{Introduction}

The unique structure and properties of propellanes have fascinated chemists for the last 50 years. ${ }^{[1]}$ The strained molecules were named by Ginsburg et al. for their propeller-like shape. ${ }^{[2]}$ The smallest member of this group, [1.1.1]propellane (1), was the first polyatomic molecule with predictions of stability, vibrational spectrum and other properties prior to its synthesis. ${ }^{[3]}$ Wiberg et al. proved these predictions, ${ }^{[3 \mathrm{~b}, 4]}$ and enabled the study of this compound's reactivity. ${ }^{[5]}$ An improved synthesis by Szeimies et al. facilitated the access to 1, whereas the precursor 3 is readily available nowadays (Scheme 1). ${ }^{[6]}$

The compounds and subsequent polymers derived from 1 ([n]staffanes) gained interest in material applications, e.g. molecular construction sets, ${ }^{[7]}$ liquid crystals ${ }^{[8]}$ and rigid spacers. ${ }^{[9]}$ More recently, the bicyclo[1.1.1]pentyl-group found use in

[a] R. M. Bär, S. Kirschner, Prof. Dr. S. Bräse Institute of Organic Chemistry

Karlsruhe Institute of Technology (KIT)

Fritz-Haber-Weg 6, 76131 Karlsruhe, Germany

E-mail: braese@kit.edu

[b] Dr. M. Nieger

Department of Chemistry

University of Helsink

P.O. Box 55 (A. I. Virtasen aukio 1)

00014 Unversity of Helsinki, Finland

[c] Prof. Dr. S. Bräse

Institute of Toxicology and Genetics

Karlsruhe Institute of Technology (KIT)

Herman-von-Helmholtz-Platz 1, 76344 Eggenstein-Leopoldshafen,

Germany

Supporting information for this article is given via a link at the end of the document medicinal chemistry as bioisoster of para-substituted benzene ${ }^{[10]}$ and alkyne moieties. ${ }^{[1]}$ With similar length and angle proportions, the rigid group can alter the physicochemical properties such as solubility and permeability and increase the three-dimensionality. Driven by the exploration of novel chemical space for drug development, Bunker et al. developed a facile route to bicyclo[1.1.1]pentylamine, a useful building block, in four steps from 1. ${ }^{[12]}$ The group of Baran reported a method for direct "propellerization" of amines by strain-release amination. ${ }^{[13]}$ This click-like reaction ${ }^{[14]}$ enables the late-stage modification of amines via the turbo-amides.

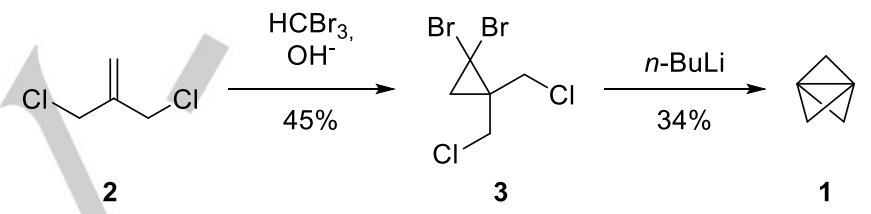

Scheme 1. Synthesis of [1.1.1]propellane (1) by Szeimies et al.[6]

The reaction of [1.1.1]propellanes like 4 with thiophenol (6a) occurs rapidly, as Szeimies et al. were the first to mention in 1985 (Scheme 2). ${ }^{[6]}$ The reaction of $\mathbf{1}$ with $\mathbf{6 a}$ became a method for quantification of 1 in solution, given the high yield. ${ }^{[5,15]}$ However, no systematic investigation of the thiol addition to 1 has been published so far. ${ }^{[5-7,15-16]}$ For example, only thiophenyl itself was used as thiol moiety. The addition of thiols to similar systems, e.g. housanes, has already illustrated the importance of such modifications. ${ }^{[17]}$

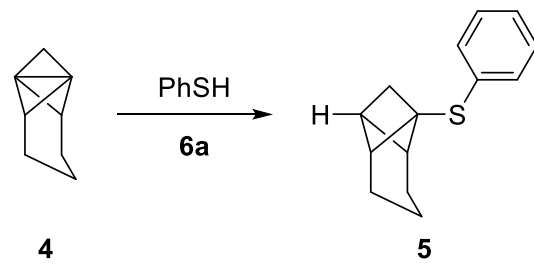

Scheme 2. First addition of thiophenol (6a) to a [1.1.1]propellane (4).[6]

In this study we added different thiols to $\mathbf{1}$ in order to obtain bicyclo[1.1.1]pentylsulfides in simple reactions. These reactions occurred at room temperature in short time without any catalysts and showed good functional group tolerance. Further modification of the thioethers enable a variety of applications, e.g. in surface or peptide modifications or as novel building blocks in medicinal chemistry.

\section{Results and Discussion}

The preparation of $\mathbf{1}$ was performed from cyclopropane $\mathbf{3}$ with methyllithium in pentane/diethyl ether according to the optimized 
procedure by Mondanaro and Dailey. ${ }^{[15]}$ By vacuum distillation of the volatiles into a cooling trap, a salt-free solution of $\mathbf{1}$ and methyl bromide was obtained. In some reactions the methyl bromide led to by-products. In this cases the procedure by Baran et al. was used to synthesize a pure stock solution of $\mathbf{1}$ with phenyllithium. ${ }^{[13]}$ As mentioned previously, the reaction of $\mathbf{1}$ with thiophenol (6a) occurs quantitatively and was used to quantify the amount of $\mathbf{1}$ in the solution. With this procedures yields of $49-73 \%$ (based on 3 ) were obtained.

The highly strained central bond of $\mathbf{1}$ is known to react with free radicals. ${ }^{[5]}$ The resulting bicyclo[1.1.1]pentyl radical is very stable compared to the corresponding cation. This is the reas on for very few by-products in this kind of reaction. ${ }^{[18]}$ It is already known that 1 inserts into disulfide bonds. ${ }^{[5]}$ The very fast addition of thiophenoxy radicals to 1 suggest a similar mechanism for the thiol addition. ${ }^{[19]}$

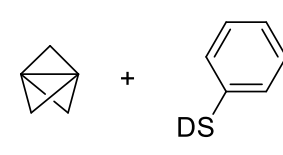

1

$6 \mathbf{a}_{d}$

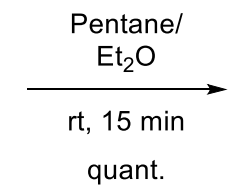

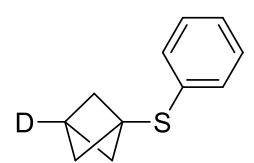

$7 \mathbf{a}_{d}$
Scheme 3. Addition of deuterated thiophenol $\left(6 a_{d}\right)$ to 1 . The central $C-C$ bond opens to form the bicyclo[1.1.1]pentane $7 \mathrm{a}_{d}$.

As a first step towards the elucidation of the mechanism of the thiol addition to $\mathbf{1}$, we performed the reaction with deuterated thiophenol $\left(6 \mathbf{a}_{d}\right)$ (Scheme 3). The ${ }^{1} \mathrm{H}$ NMR spectrum of the product $7 \mathbf{a}_{d}$ showed a decreased signal for the bridgehead proton by $85 \%$ (ESI S5). With regards to the $85 \%$ deuterated thiol $6 \mathbf{a}_{d}$ (ESI S4), this indicates the expected opening of the central CC bond. The signals of the $\mathrm{CH}_{2}$ groups show a slight upfield shift for the deuterated compound $7 \mathrm{a}_{d \cdot}$. In the ${ }^{13} \mathrm{C}$ NMR spectrum both the signals for the bridgehead carbon and the quaternary carbon of the bicyclo[1.1.1]pentane disappear almost completely (ESI S6). The strong through space orbital communication between these two atoms have been known for a long time. ${ }^{[16 b, 20]}$

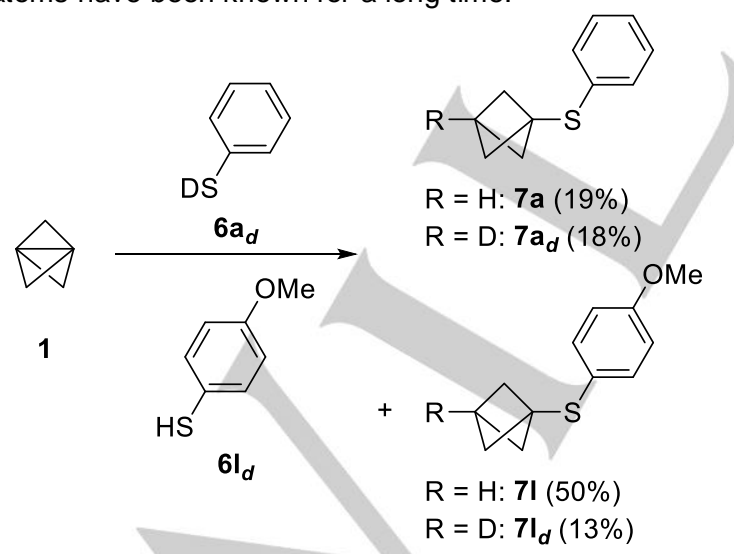

Scheme 4. Competitive reaction of deuterated thiophenol $\left(6 \mathbf{a}_{d}\right)$ and 4-methoxythiophenol (6I) with 1 . The reaction was performed in $\mathrm{Et}_{2} \mathrm{O}$ for $15 \mathrm{~min}$ at room temperature.

Szeimies et al. already proposed a radical chain mechanism for this reaction. ${ }^{[6]}$ This would request a bicyclo[1.1.1]pentyl radical $7_{r}$ that could be trapped by another thiol proton. To get a deeper insight into the reaction pathway we performed a competitive reaction of $\mathbf{1}$ with $\mathbf{6} \mathbf{a}_{d}$ and the para-methoxy derivative of thiophenol 6I (Scheme 4). We obtained a mixture of four deuterated and non-deuterated products (ESI S7). The existence of $7 \mathbf{I}_{d}$ supports the proposal of a bicyclo[1.1.1]pentyl radical $7_{r}$ as it cannot be formed by an insertion of $\mathbf{1}$ into $\mathbf{6 I}$ (Scheme 5). To exclude a proton-deuterium exchange of the products $7 \mathrm{a}_{d}$ and $7 \mathrm{I}$ we stirred these compounds together in diethyl ether for two days. No deuterated $\mathbf{7 I}_{d}$ could be observed in this mixture (ESI S8).
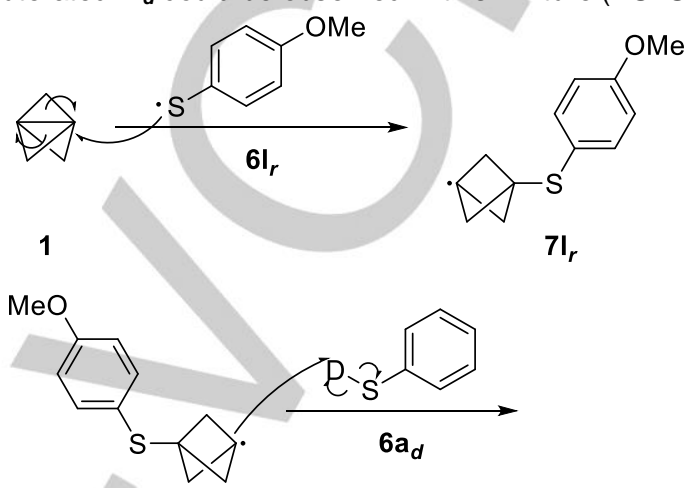

$7 I_{r}$

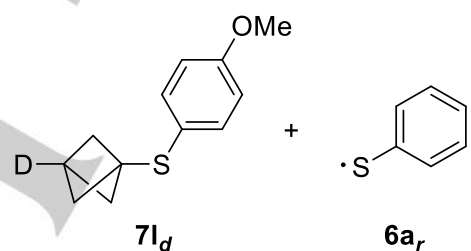

Scheme 5. Proposed reaction pathway for the formation of $7 \mathbf{I}_{d}$.

To investigate the scope and the tolerated functional groups in this reaction, different thiols were added to 1 . Therefore, a stock solution of the according thiol was added in a slight excess to the propellane solution. After $15 \mathrm{~min}$ of stirring at room temperature, the reaction was finished. Contrary to the reported addition of $6 \mathbf{a}$ to 1 where the reaction mixture was irradiated with visible light, the reaction also proceeds in the dark. ${ }^{[5]}$ The remaining thiol was removed by washing with $\mathrm{NaOH}$-solution and the solvent was removed in vacuo. If necessary, the product was purified via column chromatography, but in most cases the product was obtained purely. The results of the thiol addition are divided into aromatic thiols (Table 1), aliphatic thiols (Table 2) and dithiols (Table 3).

Monohalogenated aromatic thiols led to the desired product in moderate to very good yields (Table 1, Entries 2-5), whereas dihalogenated thiols only showed fair yields (Entries 6-7). However, it is noteworthy that halides are tolerated in this reaction and no by-products were detected at all. Alkyl-substituted aromatic thiols reacted very well with $\mathbf{1}$, except for $\mathbf{6 h}$ which only led to $57 \%$ yield (Entries 8-11). No by-products were obtained for neither the alkyl-substituted nor the 2-hydroxy-substituted product $7 \mathrm{~m}$. The latter gave a yield of $61 \%$ (Entry 13 ).

In the case of activated aromatic thiols like 6I (Entry 12), a nucleophilic substitution of methyl bromide in the stock solution of 1 took place. This led to the methylation of the thiols and therefore decreased the yield of the desired products. To verify this 
observation, a solution of $\mathbf{1}$ and methyl bromide was treated with an excess of $\mathbf{6 a}$ first to remove the propellane. After $15 \mathrm{~min}, \mathbf{6 I}$ was added and the solution was stirred for further $15 \mathrm{~min}$. After washing of the organic solution and removing the solvent, the two expected products $7 \mathrm{a}$ and methylated $6 \mathrm{I}$ were obtained.

To overcome this issue in the thiol addition, two equivalents of the thiols were used and the products could be separated in most cases by column chromatography. To avoid the formation of the by-product in order to simplify the purification, $\mathbf{1}$ was prepared with phenyllithium according to Baran et al. ${ }^{[13]}$ With no methyl bromide in the stock solution, the sulfides were obtained purely after one washing step.
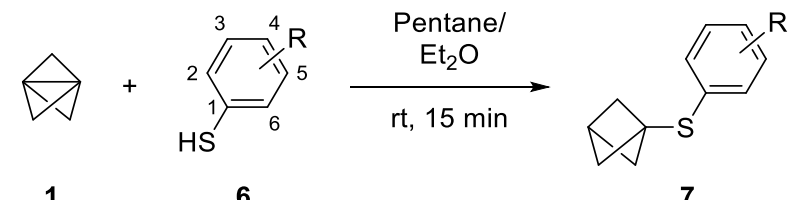

Table 1. Results of the addition of aromatic thiols 6 to 1 .

\begin{tabular}{llcccc}
\multicolumn{6}{l}{ Table 1. Results of the addition of aromatic thiols 6 to 1.} \\
\hline Entry Thiol & $R$ & $\begin{array}{c}\text { Stock solution } \\
\text { of thiol }\end{array}$ & Product & Yield[a] [\%] \\
\hline
\end{tabular}

\begin{tabular}{|c|c|c|c|c|c|}
\hline 1 & $6 a$ & $\mathrm{H}$ & $1 \mathrm{M}$ in $\mathrm{Et}_{2} \mathrm{O}$ & $7 a$ & quant. ${ }^{[b]}$ \\
\hline 2 & $6 b$ & $2-\mathrm{Cl}$ & $1 \mathrm{M}$ in $\mathrm{Et}_{2} \mathrm{O}$ & $7 b$ & 58 \\
\hline 3 & $6 c$ & $3-\mathrm{Cl}$ & $1 \mathrm{M}$ in $\mathrm{Et}_{2} \mathrm{O}$ & $7 c$ & 72 \\
\hline 4 & $6 d$ & $4-\mathrm{Cl}$ & $1 \mathrm{M}$ in $\mathrm{Et}_{2} \mathrm{O}$ & $7 d$ & 87 \\
\hline 5 & $6 e$ & $4-\mathrm{Br}$ & $1 \mathrm{M}$ in $\mathrm{Et}_{2} \mathrm{O}$ & $7 e$ & 65 \\
\hline 6 & $6 f$ & $2,6-\mathrm{Cl}_{2}$ & $\begin{array}{c}1 \mathrm{M}^{\text {in }} \mathrm{Et}_{2} \mathrm{O} / \\
\text { neat }\end{array}$ & $7 f$ & 6 \\
\hline 7 & $6 \mathrm{~g}$ & $3,5-\mathrm{Cl}_{2}$ & $1 \mathrm{M}$ in $\mathrm{Et}_{2} \mathrm{O}$ & $7 g$ & 60 \\
\hline 8 & $6 h$ & 2-Me & $1 \mathrm{M}$ in $\mathrm{Et}_{2} \mathrm{O}$ & $7 \mathrm{~h}$ & 57 \\
\hline 9 & $6 i$ & 4-Me & $1 \mathrm{M}$ in $\mathrm{Et}_{2} \mathrm{O}$ & $7 \mathbf{i}$ & 87 \\
\hline 10 & $6 j$ & $2,4,6-\mathrm{Me}_{3}$ & $1 \mathrm{M}$ in $\mathrm{Et}_{2} \mathrm{O}$ & $7 \mathrm{j}$ & 84 \\
\hline 11 & $6 k$ & 4-tBu & $1 \mathrm{M}$ in $\mathrm{Et}_{2} \mathrm{O}$ & $7 k$ & 79 \\
\hline 12 & 61 & 4-OMe & $1 \mathrm{M}$ in $\mathrm{Et}_{2} \mathrm{O}$ & 71 & 90 \\
\hline 13 & $6 m$ & $2-\mathrm{OH}$ & $1 \mathrm{M}$ in $\mathrm{Et}_{2} \mathrm{O}$ & $7 m$ & 61 \\
\hline 14 & $6 n$ & $3-\mathrm{NH}_{2}$ & $1 \mathrm{M}$ in $\mathrm{Et}_{2} \mathrm{O}$ & $7 n$ & 64 \\
\hline 15 & 60 & $4-\mathrm{NH}_{2}$ & $1 \mathrm{M}$ in $\mathrm{Et}_{2} \mathrm{O}$ & 70 & 16 \\
\hline 16 & $6 p$ & $4-\mathrm{NO}_{2}$ & $0.5 \mathrm{M}$ in $\mathrm{THF}$ & $7 p$ & 47 \\
\hline 17 & $6 q$ & $2-\mathrm{COOH}$ & neat & $7 q$ & $30[c]$ \\
\hline 18 & $6 r$ & $3-\mathrm{COOH}$ & neat & $7 r$ & $53^{[c]}$ \\
\hline
\end{tabular}

[a] Isolated yield. [b] Based on literature. ${ }^{[5]}[\mathrm{c}] 1 \mathrm{~h}$ reaction time.
Amino, nitro and carboxyl groups were also tolerated in the reaction (Entries 14-18). However, the yields decreased significantly with these thiols. Para- and ortho-substituted aromatic thiols led to the lowest yields. As no large amounts of by-products could be detected, we suppose that these products are unstable. When stored at room temperature the amines $\mathbf{7 n}$ and 70 decomposed after few days.

The carboxylic acids $\mathbf{6 q}$ and $\mathbf{6 r}$ did not dissolve in the nonpolar solvents used in this reaction. To our surprise, the neat acids without any solvent reacted with $\mathbf{1}$ in the usual stock solution to achieve the desired product. The reaction time increased to $1 \mathrm{~h}$ for the neat thiols. We suppose that to some extent, the acids dissolve in diethyl ether and whenever the thiol reacts with 1 further acid can dissolve in solution. With this discovery, we repeated the synthesis of $7 \mathrm{f}$ without dissolving the thiol prior to the addition of $\mathbf{1}$. We obtained the product in similar yields.

For the products $7 q$ and $7 r$ we were able to grow single crystals and determine the structure via X-ray diffraction (Figure 1). So far, only a few crystal structures of bicyclo[1.1.1]pentylsulfides were published and none of them contained a monosulfide with a terminal bicyclopentane moiety. ${ }^{[16 c, 21]}$ Both carboxylic acids form dimers and the bicyclo[1.1.1]pentyl-group shows a very short distance between the two bridgehead carbons of $1.859 \AA$ for $7 q$ and $1.852 \AA$ for 7 r, respectively.

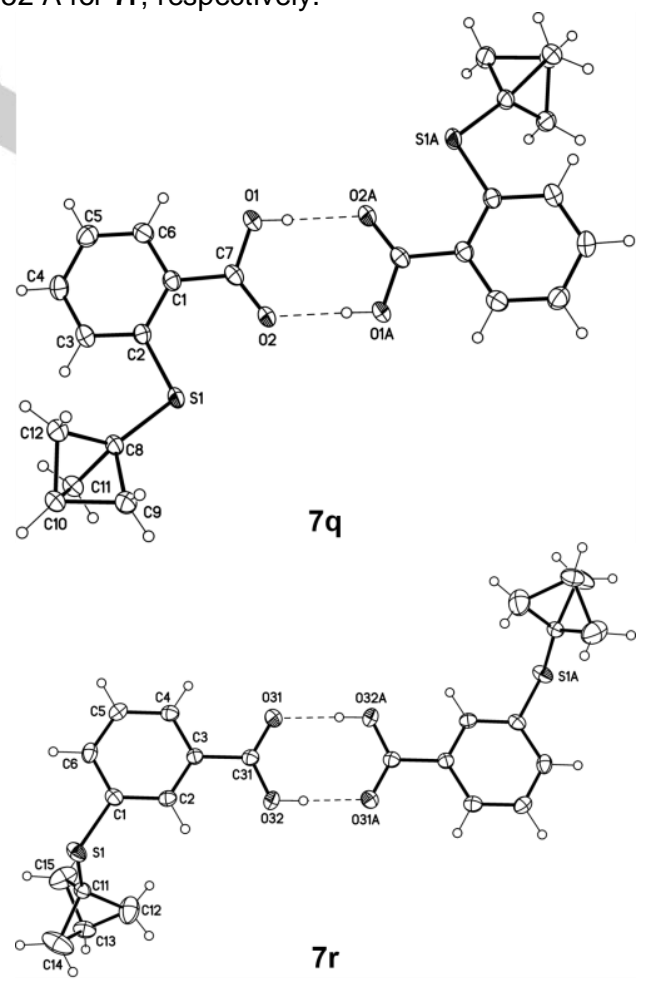

Figure 1. Structures of $7 q$ and $7 r$ dimers determined by single crystal X-ray diffraction.

Aliphatic thiols $\mathbf{8}$ also reacted with $\mathbf{1}$ without the formation of any by-product (Table 2). Simple alkyl thiols such as $\mathbf{8 a - d}$ reacted with 1 in poor to very good yields (Entries 1-4), whereas the branched alkyl thiols yielded lower than the corresponding linear alkyl thiols. It has to be noticed here, that these small compounds 
are volatile and the isolated yields varied in repeated reactions. The addition of 2-mercaptoethanol (8e) and thioglycolic acid (8f) to 1 worked well with $77 \%$ and $66 \%$ yield, respectively, and demonstrated the tolerance of hydroxyl and carboxyl groups for alkyl thiols (Entry 5-6). Benzyl mercaptane $(\mathbf{8 g})$ reacted with 1 in $53 \%$ yield (Entry 7 ). Unfortunately, a debenzylation of $9 \mathrm{~g}$ to bicyclo[1.1.1]pentylthiol was not successful, probably due to a rearrangement to a cyclobutane similar to the known rearrangement of $\mathbf{1}$ with acids ${ }^{[4 \mathrm{a}]}$ and subsequent hydrogenation. One of the most interesting thiols was the protected cysteine $\mathbf{8 h}$. The addition of this compound to 1 enables peptide modifications and leads to the new unnatural amino acid 9 h (Entry 8 ).

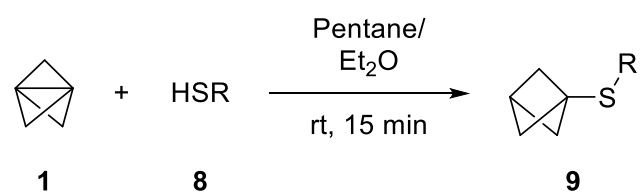

Table 2. Results of the addition of aliphatic thiols 8 to 1

\begin{tabular}{|c|c|c|c|c|c|}
\hline Entry & Thiol & $\mathrm{R}$ & $\begin{array}{l}\text { Stock } \\
\text { solution } \\
\text { of thiol }\end{array}$ & Product & $\begin{array}{c}\text { Yield[a] }^{[a]} \\
{[\%]}\end{array}$ \\
\hline 1 & $8 a$ & $n \mathrm{Pr}$ & $\begin{array}{c}1 \mathrm{M} \text { in } \\
\mathrm{Et}_{2} \mathrm{O}\end{array}$ & $9 a$ & 81 \\
\hline 2 & $8 b$ & iPr & $\begin{array}{l}1 \mathrm{M} \text { in } \\
\mathrm{Et}_{2} \mathrm{O}\end{array}$ & $9 b$ & 35 \\
\hline 3 & $8 c$ & $n B u$ & $\begin{array}{l}1 \mathrm{M} \text { in } \\
\mathrm{Et}_{2} \mathrm{O}\end{array}$ & $9 c$ & 67 \\
\hline 4 & $8 d$ & $t \mathrm{Bu}$ & $\begin{array}{c}1 \mathrm{M} \text { in } \\
\mathrm{Et}_{2} \mathrm{O}\end{array}$ & $9 c$ & \\
\hline 5 & $8 e$ & $\left(\mathrm{CH}_{2}\right)_{2} \mathrm{OH}$ & $\begin{array}{c}1 \mathrm{M} \text { in } \\
\mathrm{Et}_{2} \mathrm{O}\end{array}$ & 侟 & \\
\hline 6 & $8 f$ & $\mathrm{CH}_{2} \mathrm{CO}_{2} \mathrm{H}$ & $\begin{array}{c}1 \mathrm{M} \text { in } \\
\mathrm{Et}_{2} \mathrm{O}\end{array}$ & $9 f$ & \\
\hline 7 & $8 g$ & $\mathrm{Bn}$ & $\begin{array}{c}1 \mathrm{M} \text { in } \\
\mathrm{Et}_{2} \mathrm{O}\end{array}$ & $9 g$ & 53 \\
\hline 8 & $8 \mathrm{~h}$ & & $\begin{array}{l}0.1 \mathrm{M} \text { in } \\
\mathrm{THF}\end{array}$ & $9 \mathrm{~h}$ & 45 \\
\hline
\end{tabular}

[a] Isolated yield.

In the case of hydrogen sulfide (10) irradiation with UV-light $(254 \mathrm{~nm})$ was necessary to initiate the radical formation (Scheme 6). In a first thiol addition, bicyclo[1.1.1]pentylthiol was formed, which reacted with a second equivalent to form the disubstituted product 11 with $64 \%$ yield.

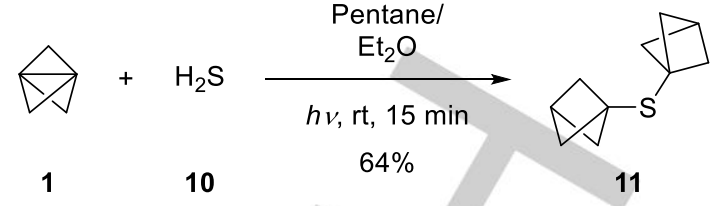

Scheme 6. Addition of hydrogen sulfide (10) $(0.8 \mathrm{M}$ in THF) to 1. The reaction was initiated with UV-light (254 nm).

The same disubstitution was observed for the dithiols 12 (Table 3). The compounds 13 were formed in satisfactory yields. Longer reaction times or more equivalents of the thiols did not increase the formation of the desired products.

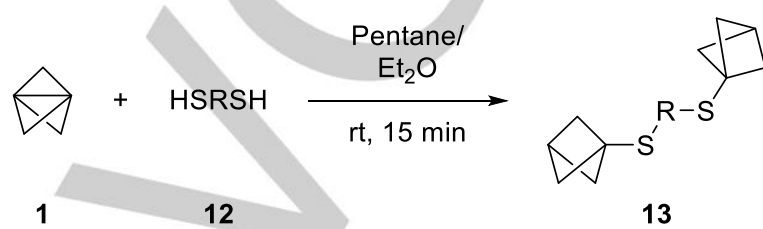

Table 3. Addition of dithiols to $\mathbf{1}$. Two equivalents of $\mathbf{1}$ were used to obtain the disubstituted products 13

\begin{tabular}{cccccc}
\hline Entry & Thiol & $\mathrm{R}$ & $\begin{array}{c}\text { Stock solution of } \\
\text { thiol }\end{array}$ & Product & Yield [a] [\%] \\
\hline 1 & $\mathbf{1 2 a}$ & $\left(\mathrm{CH}_{2}\right)_{2}$ & $1 \mathrm{M} \mathrm{in} \mathrm{Et}_{2} \mathrm{O}$ & $\mathbf{1 3 a}$ & 31 \\
\hline 2 & $\mathbf{1 2 b}$ & $\left(\mathrm{CH}_{2}\right)_{4}$ & $1 \mathrm{M} \mathrm{in} \mathrm{Et}_{2} \mathrm{O}$ & $\mathbf{1 3 b}$ & 31 \\
\hline 3 & $\mathbf{1 2 c}$ & $\left(\mathrm{CH}_{2}\right)_{6}$ & $1 \mathrm{M}$ in Et $2 \mathrm{O}$ & $\mathbf{1 3 c}$ & 47 \\
\hline
\end{tabular}

[a] Isolated yield.

For potential applications it may be necessary to further modify the products of the thiol addition to 1 . To tune the polarity oxidation is the simplest way. We performed the oxidation of $7 a$ with different amounts of $m C P B A$ to obtain the sulfoxide 14 and the sulfone $\mathbf{1 5}$ in a fast way and with a simple workup (Table 4). The yields were determined by analytical HPLC and show a smooth trend with the increasing amount of oxidizing agent.

The sulfoxide 14, which is the most polar compound of this series, is obtained in the best yield with 1.5 equivalents of $m \mathrm{CPBA}$ (Entry 3). Full oxidation of all the starting material to the sulfone 15 is achieved with 3.0 equivalents of $m C P B A$ (Entry 7). These products can be further modified, e.g. to sulfoximines, to enlarge the fields of applications. ${ }^{[22]}$

After the successful reactions of $\mathbf{1}$ with thiols we expanded our investigation towards selenols (Scheme 7). Due to the stronger nucleophilicity of selenols first attempts with a solution of $\mathbf{1}$ and methyl bromide led to the methylated by-product. With the pure stock solution of 1 the so far unknown selenobicyclus 17 was obtained in quantitative yield.

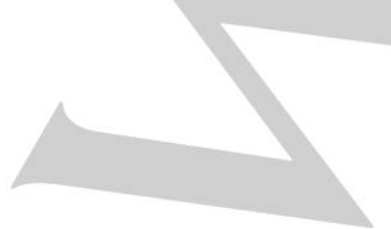

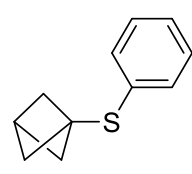

$7 a$

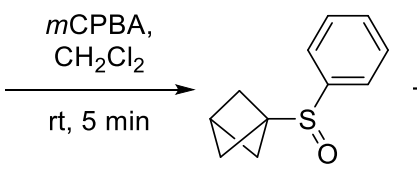

14

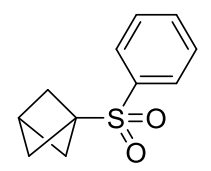

15 
Table 4. Oxidation of $7 a$ to the sulfoxide 14 and the sulfone 15.

\begin{tabular}{ccccc}
\hline Entry & Equiv. of $m$ CPBA & $\begin{array}{c}\text { Remaining } \\
\mathbf{7 a}^{[a]}[\%]\end{array}$ & $\begin{array}{c}\text { Yield 14 } \\
{[\%]}\end{array}$ & $\begin{array}{c}\text { Yield 15 } \\
{[\%]}\end{array}$ \\
\hline 1 & 1.00 & 52 & 46 & 2 \\
2 & 1.20 & 47 & 50 & 3 \\
3 & 1.50 & 15 & 70 & 15 \\
4 & 1.80 & 12 & 66 & 22 \\
5 & 2.00 & - & 43 & 57 \\
6 & 2.50 & - & 4 & 96 \\
7 & 3.00 & - & - & quant. \\
\hline
\end{tabular}

[a] Determined by analytical HPLC.

In a competitive reaction of thiophenol (6a) and benzeneselenol (16) with 1 about $80 \%$ of the selenide product 17 were obtained and only $20 \%$ of the sulfide 7 a (according to NMR, ESI S9). This indicates an even more rapid reaction of seleno-compounds with 1.

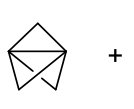<smiles>[SeH]c1ccccc1</smiles>

16

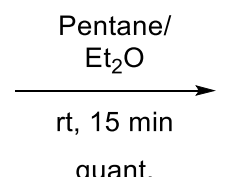

quant.

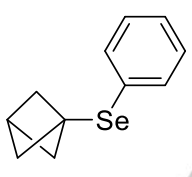

17
Scheme 7. The addition of benzeneselenol (16) to 1 afforded the product 17 in quantitative yield.

\section{Conclusions}

The thiol addition to [1.1.1]propellane is a versatile tool for many potential applications. The reaction proceeds fast, clean, without any catalyst and with no detectable amounts of by-products. Functional groups like halogens, hydroxyl, methoxy, carboxyl, amino and nitro groups are tolerated in this reaction and do not need any protection. Aromatic thiols with substituents in ortho or para position show lower yields in the addition to 1. Especially amino and nitro groups destabilize the product. For the addition of hydrogen sulfide irradiation with UV-light was necessary to generate radicals. The synthesis of the novel amino acid $\mathbf{9 h}$ enables peptide modifications that will be further studied.

Modification of the products is possible by oxidation to the corresponding sulfoxide or sulfone. The expansion of the reaction to selenols was successful and holds promise for even faster additions of such compounds. This reaction is a useful tool for modification, trapping and conjugation of thiols in many fields.

\section{Experimental Section}

For information concerning the measurements and working techniques as well as the analytical data of all other compounds, please use the supporting information. Crystallographic data can be obtained free of charge from The Cambridge Crystallographic Data Centre via www.ccdc.cam.ac.uk/data_request/cif.

[1.1.1]Propellane (1): The compound was synthesized and distilled either by general procedure $\mathbf{a}$ (with MeLi) ${ }^{[15]}$ or by general procedure $\mathbf{b}$ (with $\mathrm{PhLi}$ (ESI S2-S3). ${ }^{[13]}$ In either case, 1 was obtained in solution and stored at $-78^{\circ} \mathrm{C}$. The analytical data is in accordance with the literature. ${ }^{[4 a]}$

${ }^{1} \mathrm{H} \mathrm{NMR}\left(300 \mathrm{MHz}, \mathrm{CDCl}_{3}\right) \delta=2.00\left(\mathrm{~s}, 6 \mathrm{H}, 3 \times \mathrm{CH}_{2}\right) \mathrm{ppm} ;{ }^{13} \mathrm{C} \mathrm{NMR}$ $\left(75 \mathrm{MHz}, \mathrm{CDCl}_{3}\right) \delta=74.1\left(-, 3 \times \mathrm{CCH}_{2}\right), 1.0\left(\mathrm{C}_{\text {quart }}, 2 \times \mathrm{CCH}_{2}\right) \mathrm{ppm}$.

The concentration of 1 was determined by using the approximately quantitative reaction of $\mathbf{1}$ with $6 \mathbf{a}$ to prouct $7 \mathrm{a}$.

1-(Phenylthio)-bicyclo[1.1.1]pentane (7a): In an argon flushed $10 \mathrm{~mL}$ flask a $1 \mathrm{M}$ solution of thiol $6 \mathrm{a}$ in $\mathrm{Et}_{2} \mathrm{O}(0.68 \mathrm{~mL}, 680 \mu \mathrm{mol})$ was added to $1.0 \mathrm{~mL}$ of the stock solution of $\mathbf{1}$ (prepared by general procedure $\mathrm{a}$ or $\mathrm{b}$ ) with unknown concentration. The reaction was stirred for $15 \mathrm{~min}$ at room temperature. The mixture was diluted with $2 \mathrm{~mL} n$-pentane and washed with $1 \mathrm{M} \mathrm{NaOH}$ solution. The organic phase was dried over $\mathrm{Na}_{2} \mathrm{SO}_{4}$ and the solvent was removed under reduced pressure to obtain the product as a pale yellow oil. The turnover of this reaction is assumed to be quantitative to calculate the concentration of the solution of 1 . The analytical data is in accordance with the literature. ${ }^{[5]}$

${ }^{1} \mathrm{H}$ NMR $\left(400 \mathrm{MHz}, \mathrm{CDCl}_{3}\right): \delta=7.46-7.43(\mathrm{~m}, 2 \mathrm{H}, \mathrm{Ar}-\mathrm{H}), 7.33-7.26(\mathrm{~m}$, $3 \mathrm{H}, \mathrm{Ar}-\mathrm{H}), 2.73(\mathrm{~s}, 1 \mathrm{H}, \mathrm{CH}), 1.96\left(\mathrm{~s}, 6 \mathrm{H}, 3 \times \mathrm{CH}_{2}\right) \mathrm{ppm} ;{ }^{13} \mathrm{C} \mathrm{NMR}(100 \mathrm{MHz}$, $\left.\mathrm{CDCl}_{3}\right) \delta=134.2$ (C $\mathrm{C}$ quart, $\left.\mathrm{CAr}_{\mathrm{Ar}}\right), 133.6\left(+, 2 \times \mathrm{CH}_{\mathrm{Ar}}\right), 128.9\left(+, 2 \times \mathrm{CH}_{\mathrm{Ar}}\right)$, $127.6\left(+, \mathrm{CH}_{\mathrm{Ar}}\right), 54.1\left(-, 3 \times \mathrm{CH}_{2}\right), 45.8$ (Cquart, $\mathrm{CArSC}_{\text {Ar }}, 28.8(+, \mathrm{CH}) \mathrm{ppm}$; IR (ATR): $\tilde{v}=2998(\mathrm{~m}), 2909(\mathrm{w}), 2874(\mathrm{w}), 1583(\mathrm{w}), 1472(\mathrm{w}), 1438(\mathrm{w})$, $1203(\mathrm{~m}), 1128(\mathrm{~m}), 1088(\mathrm{w}), 1066(\mathrm{w}), 1024(\mathrm{w}), 894(w), 777(\mathrm{vw})$, $741(\mathrm{~m}), 691(\mathrm{~m}), 548(\mathrm{w}), 502(\mathrm{w}), 423(\mathrm{w}), 385(\mathrm{vw}) \mathrm{cm}^{-1}$; MS (El, $70 \mathrm{eV}): m / z(\%)=176(18)[\mathrm{M}]^{+}, 135(42)\left[\mathrm{M}-\mathrm{C}_{3} \mathrm{H}_{5}\right]^{+}, 109(71)\left[\mathrm{M}-\mathrm{C}_{5} \mathrm{H}_{7}\right]^{+}$, $99(11)\left[\mathrm{M}-\mathrm{C}_{6} \mathrm{H}_{5}\right]^{+}, 78(64)\left[\mathrm{C}_{6} \mathrm{H}_{5}+\mathrm{H}\right]^{+}, 77(39)\left[\mathrm{C}_{6} \mathrm{H}_{5}\right]^{+}, 67(97)\left[\mathrm{C}_{5} \mathrm{H}_{7}\right]^{+}, 41$ (100) $\left[\mathrm{C}_{3} \mathrm{H}_{5}\right]^{+} ; \mathrm{HRMS}(\mathrm{El}, 70 \mathrm{eV})$ : calcd for $\mathrm{C}_{11} \mathrm{H}_{12}{ }^{32} \mathrm{~S}[\mathrm{M}]^{+}$176.0654; found 176.0655 .

General procedure $\mathbf{c}$ for the thiol addition to $\mathbf{1}$ as an example for $\mathbf{7 d}$ :

Bicyclo[1.1.1]pent-1-yl(4-chlorophenyl)sulfane (7d): In an argon flushed $10 \mathrm{~mL}$ flask a $1 \mathrm{M}$ solution of thiol $6 \mathbf{d}$ in $\mathrm{Et}_{2} \mathrm{O}(0.390 \mathrm{~mL}, 390 \mu \mathrm{mol}$, 1.00 equiv.) was added to a solution of 1 ( $1.00 \mathrm{~mL}, 390 \mu \mathrm{mol}, 1.00$ equiv.) prepared by general procedure a or $\mathrm{b}$. The reaction was stirred for $15 \mathrm{~min}$ at room temperature. The mixture was diluted with $2 \mathrm{~mL} n$-pentane and washed with $1 \mathrm{M} \mathrm{NaOH}$ solution. The organic phase was dried over $\mathrm{Na}_{2} \mathrm{SO}_{4}$ and the solvent was removed under reduced pressure to obtain the product $7 \mathrm{~d}$ as a pale yellow oil in $87 \%$ yield $(71.0 \mathrm{mg}, 337 \mu \mathrm{mol})$.

${ }^{1} \mathrm{H} \mathrm{NMR}\left(400 \mathrm{MHz}, \mathrm{CDCl}_{3}\right): \delta=7.37-7.35(\mathrm{~m}, 2 \mathrm{H}, \mathrm{Ar}-\mathrm{H}), 7.28-7.26(\mathrm{~m}$, $2 \mathrm{H}, \mathrm{Ar}-\mathrm{H}), 2.73(\mathrm{~s}, 1 \mathrm{H}, \mathrm{CH}), 1.94\left(\mathrm{~s}, 6 \mathrm{H}, 3 \times \mathrm{CH}_{2}\right) \mathrm{ppm} ;{ }^{13} \mathrm{C} \mathrm{NMR}(100 \mathrm{MHz}$, $\left.\mathrm{CDCl}_{3}\right) \delta=135.0\left(+, 2 \times \mathrm{CH}_{\mathrm{Ar}}\right), 133.8\left(\mathrm{C}_{\text {quart }}, \mathrm{CAr}_{\mathrm{Ar}} \mathrm{S}\right), 132.7\left(\mathrm{C}_{\text {quart, }}, \mathrm{C}_{\mathrm{Ar}} \mathrm{Cl}\right)$, $129.1\left(+, 2 \times \mathrm{CH}_{\mathrm{Ar}}\right), 54.1\left(-, 3 \times \mathrm{CH}_{2}\right), 45.7\left(\mathrm{C}_{\text {quart }}, \mathrm{C}_{\mathrm{Ar}} \mathrm{SC}\right) \mathrm{ppm}$; IR (ATR): $\tilde{v}=2979(\mathrm{~m}), 2909(\mathrm{w}), 2874(\mathrm{w}), 1572(\mathrm{w}), 1473(\mathrm{~m}), 1387(\mathrm{w}), 1205(\mathrm{~m})$, $1127(\mathrm{~m}), 1092(\mathrm{~m}), 1012(\mathrm{~m}), 888(\mathrm{w}), 819(\mathrm{~m}), 776(\mathrm{w}), 744(\mathrm{w}), 555(\mathrm{w})$, $542(\mathrm{w}), 504(\mathrm{~m}), 449(\mathrm{w}) \mathrm{cm}^{-1}$; MS (El, $\left.70 \mathrm{eV}\right): \mathrm{m} / \mathrm{z}(\%)=212 / 210(17 / 46)$ $[\mathrm{M}]^{+}, 169(24)\left[\mathrm{M}-\mathrm{C}_{3} \mathrm{H}_{5}\right]^{+}, 144(65)\left[\mathrm{M}-\mathrm{C}_{5} \mathrm{H}_{7}+\mathrm{H}\right]^{+}, 134$ (33) $\left[\mathrm{M}-\mathrm{C}_{3} \mathrm{H}_{5}-\mathrm{Cl}\right]^{+}$, $108(37)\left[\mathrm{M}-\mathrm{C}_{5} \mathrm{H}_{7}-\mathrm{Cl}\right]^{+}, 85(31)\left[\mathrm{M}-\mathrm{C}_{6} \mathrm{H}_{4} \mathrm{Cl}-\mathrm{CH}_{3}\right]^{+}, 67(100)\left[\mathrm{C}_{5} \mathrm{H}_{7}\right]^{+} ; \mathrm{HRMS}$ (El, $70 \mathrm{eV}$ ): calcd for $\mathrm{C}_{11} \mathrm{H}_{11}{ }^{35} \mathrm{Cl}^{32} \mathrm{~S}[\mathrm{M}]+210.0270$; found: 210.0269 .

(Bicyclo[1.1.1]pent-1-yl-3-d)(phenyl)sulfane (7a $\left.a_{d}\right)$ : $7 a_{d}$ was synthesized from a solution of 1 (general procedure a) according to the general procedure $\mathrm{c}$ with $85 \%$ deuterated thiophenol $\left(6 \mathbf{a}_{d}\right)$ (ESI S3). The products $7 a_{d}(85 \%)$ and $7 \mathbf{a}(15 \%)$ were obtained as a pale yellow liquid in 
quantitative yield $(58 \mathrm{mg}, 327 \mu \mathrm{mol})$. For $7 \mathbf{a}_{d}$ the following analytical data was obtained.

${ }^{1} \mathrm{H}$ NMR $\left(400 \mathrm{MHz}, \mathrm{CDCl}_{3}\right): \delta=7.46-7.43(\mathrm{~m}, 2 \mathrm{H}, \mathrm{Ar}-\mathrm{H}), 7.33-7.26(\mathrm{~m}$, $3 \mathrm{H}, \mathrm{Ar}-\mathrm{H}), 1.95\left(\mathrm{~s}, 6 \mathrm{H}, 3 \times \mathrm{CH}_{2}\right) \mathrm{ppm} ;{ }^{13} \mathrm{C} \mathrm{NMR}\left(100 \mathrm{MHz}, \mathrm{CDCl}_{3}\right) \delta=$ 134.2 ( $\left.\mathrm{C}_{\text {quart }}, \mathrm{C}_{\mathrm{Ar}}\right), 133.6\left(+, 2 \times \mathrm{CH}_{\mathrm{Ar}}\right), 128.9\left(+, 2 \times \mathrm{CH}_{\mathrm{Ar}}\right), 127.6\left(+, \mathrm{CH}_{\mathrm{Ar}}\right)$, $54.0\left(-, 3 \times \mathrm{CH}_{2}\right), 45.8\left(\mathrm{C}_{\text {quart }}, \mathrm{C}_{\mathrm{Ar}} \mathrm{SC}\right) \mathrm{ppm}$; IR (ATR): $\tilde{v}=2980(\mathrm{w})$, $2910(w), 2875(w), 2227(v w), 1583(w), 1473(w), 1438(w), 1201(m)$, $1120(\mathrm{~m}), 1088(\mathrm{w}), 1066(\mathrm{w}), 1024(\mathrm{w}), 895(\mathrm{w}), 743(\mathrm{~m}), 691(\mathrm{~m})$, $548(\mathrm{w}), 502(\mathrm{w}), 422(\mathrm{vw}) \mathrm{cm}^{-1}$; MS (EI, $\left.70 \mathrm{eV}\right): \mathrm{m} / \mathrm{z}(\%)=177(45)[\mathrm{M}]^{+}$, 135 (74) $\left[\mathrm{M}-\mathrm{C}_{3} \mathrm{H}_{4}{ }^{2} \mathrm{H}\right]^{+}, 110(100)\left[\mathrm{M}-\mathrm{C}_{5} \mathrm{H}_{6}{ }^{2} \mathrm{H}+\mathrm{H}\right]^{+}, 109(27)\left[\mathrm{M}-\mathrm{C}_{5} \mathrm{H}_{6}{ }^{2} \mathrm{H}\right]^{+}$, $100(11)\left[\mathrm{M}-\mathrm{C}_{6} \mathrm{H}_{5}\right]^{+}, 77(14)\left[\mathrm{C}_{6} \mathrm{H}_{5}\right]^{+}, 68(61)\left[\mathrm{C}_{5} \mathrm{H}_{6}{ }^{2} \mathrm{H}\right]^{+} ; \mathrm{HRMS}(\mathrm{El}, 70 \mathrm{eV}):$ calcd for $\mathrm{C}_{11} \mathrm{H}_{11}{ }^{2} \mathrm{H}^{32} \mathrm{~S}[\mathrm{M}]^{+} 177.0717$; found 177.0715

Bicyclo[1.1.1]pent-1-yl(2-chlorophenyl)sulfane (7b): 7b was synthesized from a solution of $\mathbf{1}$ (general procedure a) according to the general procedure $\mathrm{c}$. The product $\mathbf{7 b}$ was obtained as a yellow liquid in $58 \%$ yield (91.0 mg, $432 \mu \mathrm{mol})$.

${ }^{1} \mathrm{H}$ NMR $\left(400 \mathrm{MHz}, \mathrm{CDCl}_{3}\right): \delta=7.57-7.55(\mathrm{~m}, 1 \mathrm{H}, \mathrm{Ar}-\mathrm{H}), 7.43-7.40(\mathrm{~m}$, $1 \mathrm{H}, \mathrm{Ar}-\mathrm{H}), 7.22-7.19(\mathrm{~m}, 2 \mathrm{H}, \mathrm{Ar}-\mathrm{H}), 2.75(\mathrm{~s}, 1 \mathrm{H}, \mathrm{CH}), 2.02\left(\mathrm{~s}, 6 \mathrm{H}, 3 \times \mathrm{CH}_{2}\right)$ ppm; ${ }^{13} \mathrm{C}$ NMR $\left(100 \mathrm{MHz}, \mathrm{CDCl}_{3}\right) \delta=137.1\left(\mathrm{C}_{\text {quart }}, \mathrm{C}_{\mathrm{Ar}} \mathrm{Cl}\right), 134.8\left(+, \mathrm{CH}_{\mathrm{Ar}}\right)$, $133.9\left(\mathrm{C}_{\text {quart }}, C_{\mathrm{Ar}} \mathrm{S}\right), 130.0\left(+, \mathrm{CH}_{\mathrm{Ar}}\right), 128.6\left(+, \mathrm{CH}_{\mathrm{Ar}}\right), 127.0\left(+, \mathrm{CH}_{\mathrm{Ar}}\right), 54.4$ $\left(-, \quad 3 \times \mathrm{CH}_{2}\right), 45.4\left(\mathrm{C}_{\text {quart }}, \mathrm{C}_{\mathrm{Ar}} \mathrm{SC}\right), 29.3(+, \mathrm{CH}) \mathrm{ppm}$; IR (ATR): $\tilde{v}=2959(\mathrm{w}), 2913(\mathrm{~m}), 2874(\mathrm{w}), \quad 1574(\mathrm{vw}), \quad 1449(\mathrm{~m}), 1427(\mathrm{w})$, $1375(w), 1249(w), 1205(m), 1123(w), 1035(m), 923(v w), 894(w)$, $748(\mathrm{~m}), 658(\mathrm{w}), 551(\mathrm{vw}), 463(\mathrm{vw}), 432(\mathrm{w}) \mathrm{cm}^{-1}$; MS (El, $\left.70 \mathrm{eV}\right): \mathrm{m} / \mathrm{z}$ $(\%)=212 / 210(10 / 27)[\mathrm{M}]^{+}, 169(26)\left[\mathrm{M}-\mathrm{C}_{3} \mathrm{H}_{5}\right]^{+}, 144(67)\left[\mathrm{M}-\mathrm{C}_{5} \mathrm{H}_{7}+\mathrm{H}\right]^{+}$, $134(40)\left[\mathrm{M}-\mathrm{C}_{3} \mathrm{H}_{5}-\mathrm{Cl}\right]^{+}, 108(40)\left[\mathrm{M}-\mathrm{C}_{5} \mathrm{H}_{7}-\mathrm{Cl}\right]^{+}, 85(20)\left[\mathrm{M}-\mathrm{C}_{6} \mathrm{H}_{4} \mathrm{Cl}-\mathrm{CH}_{3}\right]^{+}$, $67(100)\left[\mathrm{C}_{5} \mathrm{H}_{7}\right]^{+}$; HRMS (El, $70 \mathrm{eV}$ ): calcd for $\mathrm{C}_{11} \mathrm{H}_{11}{ }^{35} \mathrm{Cl}^{32} \mathrm{~S}[\mathrm{M}]^{+} 210.0270$; found 210.0270

Bicyclo[1.1.1]pent-1-yl(3-chlorophenyl)sulfane (7c): 7c was synthesized from a solution of $\mathbf{1}$ (general procedure b) according to the general procedure $\mathrm{c}$. The product $7 \mathrm{c}$ was obtained as a yellow liquid in $72 \%$ yield (53.0 mg, $252 \mu \mathrm{mol})$.

${ }^{1} \mathrm{H}$ NMR $\left(400 \mathrm{MHz}, \mathrm{CDCl}_{3}\right): \delta=7.44-7.43(\mathrm{~m}, 1 \mathrm{H}, \mathrm{Ar}-\mathrm{H}), 7.32-7.30(\mathrm{~m}$, $1 \mathrm{H}, \mathrm{Ar}-\mathrm{H}), 7.24-7.22(\mathrm{~m}, 2 \mathrm{H}, \mathrm{Ar}-\mathrm{H}), 2.75(\mathrm{~s}, 1 \mathrm{H}, \mathrm{CH}), 1.98\left(\mathrm{~s}, 6 \mathrm{H}, 3 \times \mathrm{CH}_{2}\right)$ ppm; ${ }^{13} \mathrm{C}$ NMR $\left(100 \mathrm{MHz}, \mathrm{CDCl}_{3}\right) \delta=136.4\left(\mathrm{C}_{\text {quart }}, C_{\mathrm{Ar}} \mathrm{Cl}\right), 134.4$ (C quart, $\left.C_{\mathrm{Ar}} \mathrm{S}\right), 132.9\left(+, \mathrm{CH}_{\mathrm{Ar}}\right), 131.3\left(+, \mathrm{CH}_{\mathrm{Ar}}\right), 129.9\left(+, \mathrm{CH}_{\mathrm{Ar}}\right), 127.6\left(+, \mathrm{CH}_{\mathrm{Ar}}\right)$, $54.2\left(-, 3 \times \mathrm{CH}_{2}\right), 45.5$ ( $\left.\mathrm{C}_{\text {quart, }} \mathrm{C}_{\mathrm{ArSC}} \mathrm{S}\right), 29.0$ (+, CH) ppm; IR (ATR): $\tilde{v}=3467(\mathrm{vw}), 2924(\mathrm{w}), 2853(\mathrm{w}), 1746(\mathrm{w}), \quad 1575(\mathrm{vw}), 1461(\mathrm{w})$, $1378(w), 1358(w), 1247(w), 1206(w), 1130(w), 1089(m), 1069(w)$, $1006(w), 939(v w), 891(w), 871(w), 853(w), 833(m), 814(w), 772(m)$, $681(\mathrm{w}), 667(\mathrm{w}), 545(\mathrm{vw}), 524(\mathrm{vw}) \mathrm{cm}^{-1}$; MS (El, $\left.70 \mathrm{eV}\right): \mathrm{m} / \mathrm{z}(\%)=$ $212 / 210(15 / 38)[\mathrm{M}]^{+}, 169(23)\left[\mathrm{M}-\mathrm{C}_{3} \mathrm{H}_{5}\right]^{+}, 144(43)\left[\mathrm{M}-\mathrm{C}_{5} \mathrm{H}_{7}+\mathrm{H}\right]^{+}, 134(37)$ $\left[\mathrm{M}-\mathrm{C}_{3} \mathrm{H}_{5}-\mathrm{Cl}\right]^{+}, 108(36)\left[\mathrm{M}-\mathrm{C}_{5} \mathrm{H}_{7}-\mathrm{Cl}\right]^{+}, 85(20)\left[\mathrm{M}-\mathrm{C}_{6} \mathrm{H}_{4} \mathrm{Cl}-\mathrm{CH}_{3}\right]^{+}, 67$ (100) [ $\left.\mathrm{C}_{5} \mathrm{H}_{7}\right]^{+}$; HRMS (EI, $70 \mathrm{eV}$ ): calcd for $\mathrm{C}_{11} \mathrm{H}_{11}{ }^{35} \mathrm{Cl}^{32} \mathrm{~S}[\mathrm{M}]^{+} 210.0270$; found 210.0271 .

Bicyclo[1.1.1]pent-1-yl(4-bromophenyl)sulfane (7e): 7e was synthesized from a solution of $\mathbf{1}$ (general procedure a) according to the general procedure $\mathrm{c}$. The product $7 \mathrm{e}$ was obtained as a yellow liquid in $65 \%$ yield (45.0 mg, $176 \mu \mathrm{mol})$.

${ }^{1} \mathrm{H}$ NMR $\left(500 \mathrm{MHz}, \mathrm{CDCl}_{3}\right): \delta=7.44-7.41(\mathrm{~m}, 2 \mathrm{H}, \mathrm{Ar}-\mathrm{H}), 7.31-7.28(\mathrm{~m}$, $2 \mathrm{H}, \mathrm{Ar}-\mathrm{H}), 2.73(\mathrm{~s}, 1 \mathrm{H}, \mathrm{CH}), 1.95\left(\mathrm{~s}, 6 \mathrm{H}, 3 \times \mathrm{CH}_{2}\right) \mathrm{ppm} ;{ }^{13} \mathrm{C} \mathrm{NMR}(125 \mathrm{MHz}$ $\left.\mathrm{CDCl}_{3}\right) \delta=135.2\left(+, 2 \times \mathrm{CH}_{\mathrm{Ar}}\right), 133.4\left(\mathrm{C}_{\text {quart }}, \mathrm{CArS}_{\mathrm{Ar}}\right), 132.0\left(+, 2 \times \mathrm{CH}_{\mathrm{Ar}}\right)$, 121.9 ( $\left.\mathrm{C}_{\text {quart }}, \mathrm{C}_{\mathrm{Ar}} \mathrm{Br}\right), 54.1\left(-, 3 \times \mathrm{CH}_{2}\right), 45.6\left(\mathrm{C}_{\text {quart }}, \mathrm{C}_{\mathrm{Ar}} \mathrm{SC}\right), 28.9(+, \mathrm{CH})$ ppm; IR (ATR): $\tilde{v}=2979(\mathrm{~m}), 2910(\mathrm{w}), 2875(\mathrm{w}), 1561(\mathrm{w}), 1471(\mathrm{~m})$, $1384(\mathrm{w}), 1205(\mathrm{~m}), 1128(\mathrm{~m}), 1090(\mathrm{~m}), 1069(\mathrm{~m}), 1009(\mathrm{~m}), 895(\mathrm{~m})$, $815(\mathrm{~m}), 776(\mathrm{w}), 729(\mathrm{w}), 549(\mathrm{w}), 511(\mathrm{w}), 492(\mathrm{w}), 445(\mathrm{w}) \mathrm{cm}^{-1}$; MS (El,
$70 \mathrm{eV}): m / z(\%)=256 / 254(26 / 26)[\mathrm{M}]^{+}, 190 / 188(30 / 29)\left[\mathrm{M}-\mathrm{C}_{5} \mathrm{H}_{7}+\mathrm{H}\right]^{+}, 134$ (54) $\left[\mathrm{M}-\mathrm{C}_{3} \mathrm{H}_{5}-\mathrm{Br}\right]^{+}, 109(32)\left[\mathrm{M}-\mathrm{C}_{5} \mathrm{H}_{7}-\mathrm{Br}+\mathrm{H}\right]^{+}, 108(40)\left[\mathrm{M}-\mathrm{C}_{5} \mathrm{H}_{7}-\mathrm{Br}\right]^{+}, 85$ (28) $\left[\mathrm{M}-\mathrm{C}_{6} \mathrm{H}_{4} \mathrm{Br}-\mathrm{CH}_{3}\right]^{+}, 67$ (100) $\left[\mathrm{C}_{5} \mathrm{H}_{7}\right]^{+}$; HRMS (EI, $70 \mathrm{eV}$ ): calcd for $\mathrm{C}_{11} \mathrm{H}_{11}{ }^{79} \mathrm{Br}^{32} \mathrm{~S}[\mathrm{M}]^{+}$253.9759; found 253.9759

Bicyclo[1.1.1]pent-1-yl(2,6-dichlorophenyl)sulfane (7f): $7 f \quad$ was synthesized from a solution of $\mathbf{1}$ (general procedure a) according to the general procedure $\mathrm{c}, \mathbf{6 f}$ was either added as a $1 \mathrm{M}$ solution in $\mathrm{Et}_{2} \mathrm{O}$ or as a solid. The product $7 \mathrm{f}$ was obtained as a yellow solid in $65 \%$ yield (154 $\mathrm{mg}$, $628 \mu \mathrm{mol})$.

m.p. $40-42{ }^{\circ} \mathrm{C} ;{ }^{1} \mathrm{H} \mathrm{NMR}\left(400 \mathrm{MHz}, \mathrm{CDCl}_{3}\right) \delta=7.41\left(\mathrm{~d},{ }^{3} \mathrm{~J}=7.7 \mathrm{~Hz}, 2 \mathrm{H}\right.$, Ar-H), $7.18\left(\mathrm{dd},{ }^{3} \mathrm{~J}=7.7 \mathrm{~Hz},{ }^{3} \mathrm{~J}=7.7 \mathrm{~Hz}, 1 \mathrm{H}, \mathrm{Ar}-\mathrm{H}\right), 2.67(\mathrm{~s}, 1 \mathrm{H}, \mathrm{CH}), 1.96$ (s, $\left.3 \times \mathrm{CH}_{2}\right) \mathrm{ppm} ;{ }^{13} \mathrm{C} \mathrm{NMR}\left(100 \mathrm{MHz}, \mathrm{CDCl}_{3}\right) \delta=142.5\left(\mathrm{C}_{\text {quart }}, 2 \times \mathrm{C}_{\mathrm{Ar}} \mathrm{Cl}\right)$, $131.9\left(\mathrm{C}_{\text {quart }}, \mathrm{C}_{\mathrm{Ar}} \mathrm{S}\right), 130.2\left(+, \mathrm{CH}_{\mathrm{Ar}}\right), 128.6\left(+, 2 \times \mathrm{CH}_{\mathrm{Ar}}\right), 54.7\left(-, 3 \times \mathrm{CH}_{2}\right)$, $46.0\left(\mathrm{C}_{\text {quart }}, \mathrm{C}_{\mathrm{Ar}} \mathrm{SC}\right), 28.5(+, \mathrm{CH}) \mathrm{ppm}$; IR (ATR): $\tilde{v}=2980(\mathrm{w}), 2909(\mathrm{w})$, $2875(\mathrm{w}), 1553(\mathrm{~m}), 1422(\mathrm{~m}), 1400(\mathrm{~m}), 1202(\mathrm{~m}), 1185(\mathrm{~m}), 1123(\mathrm{~m})$ $1086(w), 926(w), 892(m), 773(s), 708(m), 557(w), 557(w), 517(w)$, 476 (w), 436 (w), 398 (w) $\mathrm{cm}^{-1}$; MS (El, $\left.70 \mathrm{eV}\right): \mathrm{m} / z(\%)=248 / 246 / 244$ $(1 / 5 / 7)[\mathrm{M}]^{+}, 209(14)[\mathrm{M}-\mathrm{Cl}]^{+}, 178(29)\left[\mathrm{M}-\mathrm{C}_{5} \mathrm{H}_{7}+\mathrm{H}\right]^{+}, 142(28)\left[\mathrm{M}-\mathrm{C}_{5} \mathrm{H}_{7}-\right.$ $\mathrm{Cl}]^{+}, 107$ (10) $\left[\mathrm{M}-\mathrm{C}_{5} \mathrm{H}_{7}-\mathrm{Cl}_{2}\right]^{+}, 85$ (22) $\left[\mathrm{M}-\mathrm{C}_{6} \mathrm{H}_{3} \mathrm{Cl}_{2}-\mathrm{CH}_{2}\right]^{+}, 67$ (100) [M$\left.\mathrm{C}_{6} \mathrm{H}_{3} \mathrm{Cl}_{2} \mathrm{~S}\right]^{+}$; HRMS (El, $70 \mathrm{eV}$ ): calcd for $\mathrm{C}_{11} \mathrm{H}_{10}{ }^{35} \mathrm{Cl}_{2}{ }^{32} \mathrm{~S}[\mathrm{M}]^{+} 243.9875$; found 243.9876 .

Bicyclo[1.1.1]pent-1-yl(3,5-dichlorophenyl)sulfane (7g): $\quad 7 g \quad$ was synthesized from a solution of $\mathbf{1}$ (general procedure a) according to the general procedure $\mathrm{c}$. The product $\mathbf{7 g}$ was obtained as a yellow liquid in $60 \%$ yield $(39.0 \mathrm{mg}, 159 \mu \mathrm{mol})$.

${ }^{1} \mathrm{H}$ NMR $\left(400 \mathrm{MHz}, \mathrm{CDCl}_{3}\right) \delta=7.30\left(\mathrm{~d},{ }^{3} \mathrm{~J}=1.8 \mathrm{~Hz}, 2 \mathrm{H}, \mathrm{Ar}-\mathrm{H}\right), 7.25(\mathrm{t}$, $\left.{ }^{3} \mathrm{~J}=1.8 \mathrm{~Hz}, 1 \mathrm{H}, \mathrm{Ar}-\mathrm{H}\right), 2.78(\mathrm{~s}, 1 \mathrm{H}, \mathrm{CH}), 2.02\left(\mathrm{~s}, 6 \mathrm{H}, 3 \times \mathrm{CH}_{2}\right) \mathrm{ppm}$; ${ }^{13} \mathrm{C} \mathrm{NMR}\left(100 \mathrm{MHz}, \mathrm{CDCl}_{3}\right) \delta=138.1\left(\mathrm{C}_{\text {quart }}, C_{\mathrm{Ar}} \mathrm{S}\right), 134.9$ (C quart, $\left.2 \times C_{\mathrm{Ar}} \mathrm{Cl}\right), 130.7\left(+, 2 \times \mathrm{CH}_{\mathrm{Ar}}\right), 127.5\left(+, \mathrm{CH}_{\mathrm{Ar}}\right), 54.4\left(-, 3 \times \mathrm{CH}_{2}\right), 45.2$ (C quart, $\left.\mathrm{C}_{\mathrm{Ar}} \mathrm{SC}\right), 29.3(+, \mathrm{CH}) \mathrm{ppm}$; IR (ATR): $\tilde{v}=2981(\mathrm{~m}), 2908(\mathrm{w})$, $2876(\mathrm{w}), 1554(\mathrm{~s}), 1401(\mathrm{~m}), 1379(\mathrm{w}), 1206(\mathrm{~m}), 1141(\mathrm{~m}), 1125(\mathrm{~m})$, $1100(\mathrm{~m}), 894(\mathrm{w}), 854(\mathrm{~m}), 795$ (s), $670(\mathrm{~m}), 428(\mathrm{w}) \mathrm{cm}^{-1}$; MS (El, $\left.70 \mathrm{eV}\right)$ : $\mathrm{m} / \mathrm{z}(\%)=248 / 246 / 244(2 / 10 / 15)\left[\mathrm{M}^{+}, 178(20)\left[\mathrm{M}-\mathrm{C}_{5} \mathrm{H}_{7}+\mathrm{H}\right]^{+}, 142(47)[\mathrm{M}-\right.$ $\left.\mathrm{C}_{5} \mathrm{H}_{7}-\mathrm{Cl}\right]^{+}, 107$ (12) $\left[\mathrm{M}-\mathrm{C}_{5} \mathrm{H}_{7}-\mathrm{Cl}_{2}\right]^{+}, 99(36)\left[\mathrm{C}_{5} \mathrm{H}_{7} \mathrm{~S}\right]^{+}, 85$ (14) $\left[\mathrm{M}-\mathrm{C}_{6} \mathrm{H}_{3} \mathrm{Cl}_{2}-\right.$ $\left.\mathrm{CH}_{3}\right]^{+}, 67$ (100) [M-C6 $\left.\mathrm{H}_{3} \mathrm{Cl}_{2} \mathrm{~S}\right]^{+}$; HRMS (EI, $70 \mathrm{eV}$ ): calcd for $\mathrm{C}_{11} \mathrm{H}_{10}{ }^{35} \mathrm{Cl}_{2}{ }^{32} \mathrm{~S}$ $[\mathrm{M}]^{+}$243.9875; found 243.9874

Bicyclo[1.1.1]pent-1-yl(o-tolyl)sulfane (7h): $7 \mathrm{~h}$ was synthesized from a solution of $\mathbf{1}$ (general procedure a) according to the general procedure c. The product $7 \mathrm{~h}$ was obtained as a pale yellow liquid in $57 \%$ yield $(28.0 \mathrm{mg}$, $147 \mu \mathrm{mol})$.

${ }^{1} \mathrm{H}$ NMR $\left(400 \mathrm{MHz}, \mathrm{CDCl}_{3}\right) \delta=7.48-7.46(\mathrm{~m}, 1 \mathrm{H}, \mathrm{Ar}-\mathrm{H}), 7.24-7.12(\mathrm{~m}, 3 \mathrm{H}$ $\mathrm{Ar}-\mathrm{H}), 2.70(\mathrm{~s}, 1 \mathrm{H}, \mathrm{CH}), 2.43\left(\mathrm{~s}, 3 \mathrm{H}, \mathrm{CH}_{3}\right), 1.94\left(\mathrm{~s}, 6 \mathrm{H}, 3 \times \mathrm{CH}_{2}\right) \mathrm{ppm}$; ${ }^{13} \mathrm{C}$ NMR $\left(100 \mathrm{MHz}, \mathrm{CDCl}_{3}\right) \delta=141.0\left(\mathrm{C}_{\text {quart }}, \mathrm{CArCH}_{3}\right), 135.0\left(+, \mathrm{CH}_{\mathrm{Ar}}\right)$, 133.5 (Cquart, $\left.C_{\text {ArS }}\right), 130.4\left(+, \mathrm{CH}_{\text {Ar }}\right), 127.9\left(+, \mathrm{CH}_{\text {Ar }}\right), 126.3\left(+, \mathrm{CH}_{\mathrm{Ar}}\right), 54.2$ $\left(-, 3 \times \mathrm{CH}_{2}\right), 45.8$ ( $\mathrm{C}$ quart, $\mathrm{C}_{\mathrm{ArSC}}$ ), $29.0(+, \mathrm{CH}), 21.3\left(+, \mathrm{CH}_{3}\right) \mathrm{ppm}$; IR $(A T R): \tilde{v}=2977(m), 2909(w), 2875(w), 1589(v w), 1469(w), 1378(w)$, $1203(\mathrm{~m}), 1127(\mathrm{~m}), 1061(\mathrm{w}), 1035(\mathrm{w}), 923(\mathrm{vw}), 896(\mathrm{~m}), 779(\mathrm{vw})$, $746(\mathrm{~s}), 712(\mathrm{~m}), 678(\mathrm{w}), 556(\mathrm{vw}), 460(\mathrm{w}), 424(\mathrm{w}) \mathrm{cm}^{-1}$; MS (El, $\left.70 \mathrm{eV}\right)$ : $\mathrm{m} / \mathrm{z}(\%)=190(10)[\mathrm{M}]^{+}, 149(100)\left[\mathrm{M}-\mathrm{C}_{3} \mathrm{H}_{5}\right]^{+}, 124(17)\left[\mathrm{M}-\mathrm{C}_{5} \mathrm{H}_{7}+\mathrm{H}\right]^{+}, 91$ (30) $\left[\mathrm{M}-\mathrm{C}_{5} \mathrm{H}_{7} \mathrm{~S}\right]^{+}, 67(18)\left[\mathrm{C}_{5} \mathrm{H}_{7}\right]^{+}$; HRMS (El, $\left.70 \mathrm{eV}\right)$ : calcd for $\mathrm{C}_{12} \mathrm{H}_{14}{ }^{32} \mathrm{~S}$ $[\mathrm{M}]^{+}$190.0811; found 190.0809 .

Bicyclo[1.1.1]pent-1-yl(p-tolyl)sulfane (7i): 7i was synthesized from a solution of $\mathbf{1}$ (general procedure a) according to the general procedure $\mathrm{c}$. The product $7 \mathbf{i}$ was obtained as a yellow liquid in $87 \%$ yield $(45.0 \mathrm{mg}$, $236 \mu \mathrm{mol})$. 
${ }^{1} \mathrm{H}$ NMR $\left(500 \mathrm{MHz}, \mathrm{CDCl}_{3}\right): \delta=7.34-7.32(\mathrm{~m}, 2 \mathrm{H}, \mathrm{Ar}-\mathrm{H}), 7.12-7.10(\mathrm{~m}$, $2 \mathrm{H}, \mathrm{Ar}-\mathrm{H}), 2.71(\mathrm{~s}, 1 \mathrm{H}, \mathrm{CH}), 2.34\left(\mathrm{~s}, 3 \mathrm{H}, \mathrm{CH}_{3}\right), 1.92\left(\mathrm{~s}, 6 \mathrm{H}, 3 \times \mathrm{CH}_{2}\right) \mathrm{ppm}$; ${ }^{13} \mathrm{C} \mathrm{NMR}\left(125 \mathrm{MHz}, \mathrm{CDCl}_{3}\right): \delta=137.7\left(\mathrm{C}_{\text {quart }}, \mathrm{C}_{\mathrm{Ar}} \mathrm{CH}_{3}\right), 134.0\left(+, 2 \times \mathrm{CH}_{\mathrm{Ar}}\right)$ 130.4 ( $\left.\mathrm{C}_{\text {quart }}, \mathrm{C}_{\mathrm{Ar}} \mathrm{S}\right), 129.7\left(+, 2 \times \mathrm{CH}_{\mathrm{Ar}}\right), 54.0\left(-, 3 \times \mathrm{CH}_{2}\right), 45.9$ (C quart, $\mathrm{C}_{\mathrm{ArSC}}$ ), $28.7(+, \mathrm{CH}), 21.3\left(+, \mathrm{CH}_{3}\right) \mathrm{ppm}$; IR (ATR): $\tilde{v}=2978(\mathrm{~m}), 2910(\mathrm{w})$ $2874(w), 1490(\mathrm{~m}), 1447(\mathrm{w}), 1398(\mathrm{vw}), 1205(\mathrm{~m}), 1129(\mathrm{~m}), 1093(\mathrm{w})$, $1018(w), 890(w), 808(m), 775(w), 733(w), 706(w), 549(w), 507(m)$, $449(\mathrm{w}) \mathrm{cm}^{-1}$; MS (El, $\left.70 \mathrm{eV}\right): \mathrm{m} / \mathrm{z}(\%)=191$ (13) $[\mathrm{M}+1]^{+}, 190(87)[\mathrm{M}]^{+}$, $175(10)\left[\mathrm{M}-\mathrm{CH}_{3}\right]^{+}, 149(100)\left[\mathrm{M}-\mathrm{C}_{3} \mathrm{H}_{5}\right]^{+}, 134(32)\left[\mathrm{M}-\mathrm{C}_{3} \mathrm{H}_{5}-\mathrm{CH}_{3}\right]^{+}, 124$ (85) $\left[\mathrm{M}-\mathrm{C}_{5} \mathrm{H}_{7}+\mathrm{H}\right]^{+}, 123(30)\left[\mathrm{M}-\mathrm{C}_{5} \mathrm{H}_{7}\right]^{+}, 91(87)\left[\mathrm{M}-\mathrm{C}_{5} \mathrm{H}_{7} \mathrm{~S}\right]^{+}, 85(37)[\mathrm{M}-$ $\left.\mathrm{C}_{6} \mathrm{H}_{4} \mathrm{CH}_{3}-\mathrm{CH}_{3}\right]^{+}, 67(52)\left[\mathrm{C}_{5} \mathrm{H}_{7}\right]^{+}$; HRMS (El, $70 \mathrm{eV}$ ): calcd for $\mathrm{C}_{12} \mathrm{H}_{14}{ }^{32} \mathrm{~S}$ $[\mathrm{M}]^{+}$190.0811; found 190.0812 .

Bicyclo[1.1.1]pent-1-yl(mesityl)sulfane (7j): 7j was synthesized from a solution of 1 (general procedure a) according to the general procedure $c$. The product $7 \mathbf{j}$ was obtained as a pale yellow liquid in $84 \%$ yield $(50.0 \mathrm{mg}$, $229 \mu \mathrm{mol})$.

${ }^{1} \mathrm{H}$ NMR $\left(400 \mathrm{MHz}, \mathrm{CDCl}_{3}\right): \delta=6.95-6.92(\mathrm{~m}, 2 \mathrm{H}, \mathrm{Ar}-\mathrm{H}), 2.62(\mathrm{~s}, 1 \mathrm{H}, \mathrm{CH})$, $2.46\left(\mathrm{~s}, 6 \mathrm{H}, \mathrm{C}^{2} \mathrm{CH}_{3}+\mathrm{C}^{6} \mathrm{CH}_{3}\right), 2.27\left(\mathrm{~s}, 3 \mathrm{H}, \mathrm{C}^{4} \mathrm{CH}_{3}\right), 1.86\left(\mathrm{~s}, 6 \mathrm{H}, 3 \times \mathrm{CH}_{2}\right)$ ppm; ${ }^{13} \mathrm{C} \mathrm{NMR}\left(100 \mathrm{MHz}, \mathrm{CDCl}_{3}\right): \delta=143.6$ (Cquart. $\left.C^{2}+C^{6}\right), 138.1$ ( $\mathrm{C}_{\text {quart. }}$, $\left.C^{4}\right), 128.9\left(+, 2 \times \mathrm{CH}_{\mathrm{Ar}}\right), 128.6\left(\mathrm{C}_{\text {quart. }}, C^{1}\right), 54.2\left(-, 3 \times \mathrm{CH}_{2}\right), 46.4$ (Cquart., $\left.\mathrm{C}^{1} \mathrm{SC}\right), 28.3(+, \mathrm{CH}), 22.4\left(+, \mathrm{C}^{2} \mathrm{CH}_{3}+\mathrm{C}^{6} \mathrm{CH}_{3}\right), 21.2\left(+, \mathrm{C}^{4} \mathrm{CH}_{3}\right)$ ppm; IR (ATR): $\tilde{v}=2974(\mathrm{~m}), 2908(\mathrm{w}), 2872(\mathrm{w}), 1601(\mathrm{w}), 1448(\mathrm{w}), 1373(\mathrm{w})$ $1203(\mathrm{~m}), 1128(\mathrm{~m}), 1060(\mathrm{w}), 1030(\mathrm{w}), 894(\mathrm{w}), 848(\mathrm{~m}), 718(\mathrm{w})$, $562(\mathrm{w}), 412(\mathrm{vw}) \mathrm{cm}^{-1}$; MS (El, $\left.70 \mathrm{eV}\right): \mathrm{m} / z(\%)=218(5)[\mathrm{M}]^{+}, 203(11)$ $\left[\mathrm{M}-\mathrm{CH}_{3}\right]^{+}, 177(100)\left[\mathrm{M}-\mathrm{C}_{3} \mathrm{H}_{5}\right]^{+}, 162(35)\left[\mathrm{M}-\mathrm{C}_{3} \mathrm{H}_{5}-\mathrm{CH}_{3}\right]^{+}, 119$ (21) [M$\left.\mathrm{C}_{5} \mathrm{H}_{7} \mathrm{~S}\right]^{+}$; HRMS (EI, $70 \mathrm{eV}$ ): calcd for $\mathrm{C}_{14} \mathrm{H}_{18}{ }^{32} \mathrm{~S}[\mathrm{M}]^{+}$218.1124; found 218.1123 .

Bicyclo[1.1.1]pent-1-yl(4-(tert-butyl)phenyl)sulfane $\quad(7 \mathbf{k}): \quad 7 \mathbf{7 k}$ was synthesized from a solution of $\mathbf{1}$ (general procedure a) according to the general procedure $c$. The product $7 \mathbf{k}$ was obtained as a pale yellow liquid in $79 \%$ yield $(50.0 \mathrm{mg}, 215 \mu \mathrm{mol})$.

${ }^{1} \mathrm{H}$ NMR $\left(400 \mathrm{MHz}, \mathrm{CDCl}_{3}\right): \delta=7.38-7.35(\mathrm{~m}, 2 \mathrm{H}, \mathrm{Ar}-\mathrm{H}), 7.33-7.30(\mathrm{~m}$, $2 \mathrm{H}, \mathrm{Ar}-\mathrm{H}), 2.71(\mathrm{~s}, 1 \mathrm{H}, \mathrm{CH}), 1.95\left(\mathrm{~s}, 6 \mathrm{H}, 3 \times \mathrm{CH}_{2}\right), 1.31\left(\mathrm{~s}, 9 \mathrm{H}, 3 \times \mathrm{CH}_{3}\right)$ $\mathrm{ppm} ;{ }^{13} \mathrm{C}$ NMR $\left(100 \mathrm{MHz}, \mathrm{CDCl}_{3}\right): \delta=150.7$ (Cquart., $\left.\mathrm{CArCCH}_{3}\right), 133.4(+$, $\left.2 \times \mathrm{CH}_{\text {Ar }}\right), 130.6$ (C quart., $\left.C_{\text {Ar }} S\right), 125.9\left(+, 2 \times \mathrm{CH}_{\text {Ar }}\right), 54.1\left(-, 3 \times \mathrm{CH}_{2}\right), 45.8$ (Cquart., $\mathrm{CArSC}_{\mathrm{S}}$ ), 34.7 ( $\left.\mathrm{C}_{\text {quart. }}, \mathrm{CCH}_{3}\right), 31.4\left(+, 3 \times \mathrm{CH}_{3}\right), 28.8(+, \mathrm{CH}) \mathrm{ppm}$; IR (ATR): $\tilde{v}=2961(\mathrm{~m}), 2907(\mathrm{w}), 2873(\mathrm{w}), 1488(\mathrm{w}), 1460(\mathrm{w}), 1393(\mathrm{w})$, $1362(w), 1266(w), 1203(m), 1129(w), 1116(m), 1013(w), 895(w)$, $827(\mathrm{~m}), 742(\mathrm{w}), 561(\mathrm{~m}), 410(\mathrm{vw}) \mathrm{cm}^{-1} ; \mathrm{MS}(\mathrm{El}, 70 \mathrm{eV}): \mathrm{m} / \mathrm{z}(\%)=232$ (42) $[\mathrm{M}]^{+}, 217(100)\left[\mathrm{M}-\mathrm{CH}_{3}\right]^{+}, 151(25)\left[\mathrm{M}-\mathrm{CH}_{3}-\mathrm{C}_{5} \mathrm{H}_{7}\right]^{+}, 85$ (23) [M$\left.\mathrm{C}_{6} \mathrm{H}_{4} \mathrm{C}\left(\mathrm{CH}_{3}\right)_{3}-\mathrm{CH}_{2}\right]^{+}, 67$ (11) $\left[\mathrm{C}_{5} \mathrm{H}_{7}\right]^{+}, 57$ (27) $\left[\mathrm{C}\left(\mathrm{CH}_{3}\right)_{3}\right]^{+}$; HRMS (EI, $70 \mathrm{eV}$ ): calcd for $\mathrm{C}_{15} \mathrm{H}_{20}{ }^{32} \mathrm{~S}[\mathrm{M}]+232.1280$; found 232.1279 .

Bicyclo[1.1.1]pent-1-yl(4-methoxyphenyl)sulfane (7I): 71 was synthesized from a solution of 1 (general procedure b) according to the general procedure c. The product $7 \mathrm{I}$ was obtained as a pale yellow liquid in $90 \%$ yield $(111 \mathrm{mg}, 538 \mu \mathrm{mol})$

${ }^{1} \mathrm{H}$ NMR $\left(400 \mathrm{MHz}, \mathrm{CDCl}_{3}\right): \delta=7.40-7.35(\mathrm{~m}, 2 \mathrm{H}, \mathrm{Ar}-\mathrm{H}), 6.86-6.82(\mathrm{~m}$, $2 \mathrm{H}, \mathrm{Ar}-\mathrm{H}), 3.81\left(\mathrm{~s}, 3 \mathrm{H}, \mathrm{CH}_{3}\right), 2.69(\mathrm{~s}, 1 \mathrm{H}, \mathrm{CH}), 1.88\left(\mathrm{~s}, 6 \mathrm{H}, 3 \times \mathrm{CH}_{2}\right) \mathrm{ppm}$; ${ }^{13} \mathrm{C}$ NMR $\left(100 \mathrm{MHz}, \mathrm{CDCl}_{3}\right) \delta=159.7\left(\mathrm{C}_{\text {quart }}, \mathrm{C}_{\mathrm{Ar}} \mathrm{OCH}_{3}\right), 136.0(+$, $\left.2 \times \mathrm{CH}_{\mathrm{Ar}}\right), 124.5$ ( $\left.\mathrm{C}_{\text {quart }}, \mathrm{C}_{\mathrm{Ar}} \mathrm{S}\right), 114.5\left(+, 2 \times \mathrm{CH}_{\mathrm{Ar}}\right), 55.4\left(+, \mathrm{CH}_{3}\right), 53.8(-$, $3 \times \mathrm{CH}_{2}$ ), 46.3 ( $\mathrm{C}_{\text {quart }}, \mathrm{C}_{\mathrm{ArSC}}$ ), $28.5(+, \mathrm{CH}) \mathrm{ppm}$; IR (ATR): $\tilde{v}=2977(\mathrm{~m})$, $2908(w), 2874(w), 2835(w), 1590(m), 1571(w), 1491(s), 1462(m)$, $1440(\mathrm{w}), 1284(\mathrm{~m}), 1242(\mathrm{~s}), 1205(\mathrm{~m}), 1171(\mathrm{~m}), 1130(\mathrm{~m}), 1096(\mathrm{~m})$, $1030(\mathrm{~m}), 892(\mathrm{~m}), 826(\mathrm{~m}), 798(\mathrm{~m}), 640(\mathrm{w}), 628(\mathrm{w}), 550(\mathrm{w}), 525(\mathrm{w})$, $457(\mathrm{vw}) \mathrm{cm}^{-1}$; MS (El, $\left.70 \mathrm{eV}\right): \mathrm{m} / z(\%)=206(100)[\mathrm{M}]^{+}, 165(23)[\mathrm{M}-$ $\left.\mathrm{C}_{3} \mathrm{H}_{5}\right]^{+}, 140(66)\left[\mathrm{M}-\mathrm{C}_{5} \mathrm{H}_{7}+\mathrm{H}\right]^{+}, 139(35)\left[\mathrm{M}-\mathrm{C}_{5} \mathrm{H}_{7}\right]^{+}, 125(33)\left[\mathrm{M}-\mathrm{C}_{5} \mathrm{H}_{7}-\right.$ $\left.\mathrm{CH}_{3}+\mathrm{H}\right]^{+}, 124$ (9) $\left[\mathrm{M}-\mathrm{C}_{5} \mathrm{H}_{7}-\mathrm{CH}_{3}+\mathrm{H}\right]^{+}, 121$ (45) $\left[\mathrm{M}-\mathrm{C}_{4} \mathrm{H}_{7}-\mathrm{OCH}_{3}\right]^{+}, 85$ (20)
$\left[\mathrm{M}-\mathrm{C}_{6} \mathrm{H}_{4} \mathrm{OCH}_{3}-\mathrm{CH}_{3}\right]^{+}, 67$ (18) $\left[\mathrm{C}_{5} \mathrm{H}_{7}\right]^{+}$; HRMS (El, $70 \mathrm{eV}$ ): calcd for $\mathrm{C}_{12} \mathrm{H}_{14} \mathrm{O}^{32} \mathrm{~S}[\mathrm{M}]^{+}$206.0765; found 206.0764.

2-(Bicyclo[1.1.1]pent-1-ylthio)phenol (7m): $7 \mathrm{~m}$ was synthesized from a solution of 1 (general procedure a) according to the general procedure $\mathrm{c}$. The product $7 \mathrm{~m}$ was obtained as a pale yellow liquid in $61 \%$ yield $(91.0 \mathrm{mg}$, $473 \mu \mathrm{mol})$.

${ }^{1} \mathrm{H}$ NMR $\left(500 \mathrm{MHz}, \mathrm{CDCl}_{3}\right): \delta=7.40\left(\mathrm{dd},{ }^{3} J=7.5 \mathrm{~Hz},{ }^{4} J=1.6 \mathrm{~Hz}, 1 \mathrm{H}\right.$, Ar-H), 7.28 (ddd, ${ }^{3} J=8.2 \mathrm{~Hz},{ }^{3} \mathrm{~J}=6.3 \mathrm{~Hz},{ }^{4} \mathrm{~J}=1.6 \mathrm{~Hz}, 1 \mathrm{H}, \mathrm{Ar}-\mathrm{H}$ ), 7.00 (dd, ${ }^{3} J=8.2 \mathrm{~Hz},{ }^{4} \mathrm{~J}=1.3 \mathrm{~Hz}, 1 \mathrm{H}, \mathrm{Ar}-\mathrm{H}$ ), 6.87 (ddd, ${ }^{3} J=7.5 \mathrm{~Hz},{ }^{3} J=6.3 \mathrm{~Hz}$, $\left.{ }^{4} \mathrm{~J}=1.3 \mathrm{~Hz}, 1 \mathrm{H}, \mathrm{Ar}-\mathrm{H}\right), 6.70(\mathrm{~s}, 1 \mathrm{H}, \mathrm{OH}), 2.69(\mathrm{~s}, 1 \mathrm{H}, \mathrm{CH}), 1.87(\mathrm{~s}, 6 \mathrm{H}$, $\left.3 \times \mathrm{CH}_{2}\right)$ ppm; ${ }^{13} \mathrm{C} \mathrm{NMR}\left(125 \mathrm{MHz}, \mathrm{CDCl}_{3}\right): \delta=157.1\left(\mathrm{C}_{\text {quart. }}, \mathrm{C}_{\mathrm{Ar}} \mathrm{OH}\right)$, $136.7\left(+, \mathrm{CH}_{\mathrm{Ar}}\right), 131.4\left(+, \mathrm{CH}_{\mathrm{Ar}}\right), 120.7\left(+, \mathrm{CH}_{\mathrm{Ar}}\right), 117.4\left(\mathrm{C}_{\text {quart. }}, \mathrm{C}_{\mathrm{Ar}} \mathrm{S}\right), 114.8$ $\left(+, \mathrm{CH}_{\mathrm{Ar}}\right), 53.9\left(-, 3 \times \mathrm{CH}_{2}\right), 45.4\left(\mathrm{C}_{\text {quart. }}, \mathrm{C}_{\mathrm{Ar}} \mathrm{SC}\right), 28.3(+, \mathrm{CH}) \mathrm{ppm}$; IR (ATR): $\tilde{v}=3403(\mathrm{w}), 2979(\mathrm{~m}), 2909(\mathrm{w}), 2875(\mathrm{w}), 1573(\mathrm{w}), 1469(\mathrm{~s})$, $1342(\mathrm{w}), 1287(\mathrm{w}), 1240(\mathrm{~m}), 1202(\mathrm{~s}), 1128(\mathrm{~m}), 1026(\mathrm{~m}), 888(\mathrm{~m})$ $830(\mathrm{w}), 751(\mathrm{~s}), 680(\mathrm{w}), 527(\mathrm{w}), 475(\mathrm{~m}), 429(\mathrm{w}), 395(\mathrm{w}) \mathrm{cm}^{-1}$; MS (El, $70 \mathrm{eV}): m / z(\%)=192(5)[\mathrm{M}]^{+}, 151(100)\left[\mathrm{M}-\mathrm{C}_{3} \mathrm{H}_{5}\right]^{+}, 126(16)\left[\mathrm{M}-\mathrm{C}_{5} \mathrm{H}_{7}+\mathrm{H}\right]^{+}$, 125 (3) $\left[\mathrm{M}-\mathrm{C}_{5} \mathrm{H}_{7}\right]^{+}, 67(16)\left[\mathrm{C}_{5} \mathrm{H}_{7}\right]^{+} ;$HRMS (El, $\left.70 \mathrm{eV}\right)$ : calcd for $\mathrm{C}_{11} \mathrm{H}_{12} \mathrm{O}^{32} \mathrm{~S}[\mathrm{M}]+192.0603$; found 192.0605 .

3-(Bicyclo[1.1.1]pent-1-ylthiol)aniline (7n): $7 n$ was synthesized from a solution of 1 (general procedure a) according to the general procedure $\mathrm{c}$. The product $7 \mathrm{n}$ was obtained as a pale yellow liquid in $64 \%$ yield $(47.0 \mathrm{mg}$, $246 \mu \mathrm{mol})$.

${ }^{1} \mathrm{H}$ NMR $\left(400 \mathrm{MHz}, \mathrm{CDCl}_{3}\right): \delta=7.10-7.05(\mathrm{~m}, 1 \mathrm{H}, \mathrm{Ar}-\mathrm{H}), 6.86-6.81(\mathrm{~m}$, $1 \mathrm{H}, \mathrm{Ar}-\mathrm{H}), 6.78-6.76(\mathrm{~m}, 1 \mathrm{H}, \mathrm{Ar}-\mathrm{H}), 6.61-6.57(\mathrm{~m}, 1 \mathrm{H}, \mathrm{Ar}-\mathrm{H}), 3.65(\mathrm{~b}, 2 \mathrm{H}$, $\left.\mathrm{NH}_{2}\right), 2.72(\mathrm{~s}, 1 \mathrm{H}, \mathrm{CH}), 1.97\left(\mathrm{~s}, 6 \mathrm{H}, 3 \times \mathrm{CH}_{2}\right) \mathrm{ppm} ;{ }^{13} \mathrm{C} \mathrm{NMR}(100 \mathrm{MHz}$, $\left.\mathrm{CDCl}_{3}\right): \delta=146.7$ (C quart., $\left.C_{\mathrm{Ar}} \mathrm{NH}_{2}\right), 135.0$ (C quart., $\left.C_{\mathrm{Ar}} \mathrm{S}\right), 129.6\left(+, \mathrm{CH}_{\mathrm{Ar}}\right)$, $123.6\left(+, \mathrm{CH}_{\mathrm{Ar}}\right), 119.8\left(+, \mathrm{CH}_{\mathrm{Ar}}\right), 114.4\left(+, \mathrm{CH}_{\mathrm{Ar}}\right), 54.2\left(-, 3 \times \mathrm{CH}_{2}\right), 45.6$ (Cquart., $\left.\mathrm{C}_{\mathrm{Ar}} \mathrm{SC}\right), 28.8(+, \mathrm{CH})$ ppm; IR (ATR): $\tilde{v}=3351(\mathrm{w}), 2977(\mathrm{~m})$, $2907(w), 2873(w), 1616(m), 1588(s), 1478(m), 1438(w), 1297(w)$, $1263(w), 1204(m), 1163(w), 1127(m), 1078(w), 992(w), 886(w)$ $771(\mathrm{~m}), 687(\mathrm{~m}), 529(\mathrm{w}), 446(\mathrm{w}) \mathrm{cm}^{-1} ; \mathrm{MS}(\mathrm{El}, 70 \mathrm{eV}): \mathrm{m} / \mathrm{z}(\%)=192(6)$ $[\mathrm{M}+\mathrm{H}]^{+}, 191(49)[\mathrm{M}]^{+}, 150(26)\left[\mathrm{M}-\mathrm{C}_{3} \mathrm{H}_{5}\right]^{+}, 125(100)\left[\mathrm{M}-\mathrm{C}_{5} \mathrm{H}_{7}+\mathrm{H}\right]^{+}, 124$ (13) $\left[\mathrm{M}-\mathrm{C}_{5} \mathrm{H}_{7}\right]^{+}, 106(34)\left[\mathrm{M}-\mathrm{C}_{5} \mathrm{H}_{8}-\mathrm{NH}_{2}\right]^{+}$; HRMS (El, $70 \mathrm{eV}$ ): calcd for $\mathrm{C}_{11} \mathrm{H}_{13} \mathrm{~N}^{32} \mathrm{~S}[\mathrm{M}]^{+}$191.0763; found 191.0763

4-(Bicyclo[1.1.1]pent-1-ylthiol)aniline (7o): 70 was synthesized from a solution of 1 (general procedure $b$ ) according to the general procedure $c$. The product 70 was obtained as a yellow oil in $16 \%$ yield $(11.0 \mathrm{mg}$, $57.5 \mu \mathrm{mol})$.

${ }^{1} \mathrm{H} \mathrm{NMR}\left(400 \mathrm{MHz}, \mathrm{CDCl}_{3}\right): \delta=7.26-7.23(\mathrm{~m}, 2 \mathrm{H}, \mathrm{Ar}-\mathrm{H}), 6.63-6.61(\mathrm{~m}$, $2 \mathrm{H}, \mathrm{Ar}-\mathrm{H}), 3.70\left(\mathrm{~b}, 2 \mathrm{H}, \mathrm{NH}_{2}\right), 2.68(\mathrm{~s}, 1 \mathrm{H}, \mathrm{CH}), 1.86\left(\mathrm{~s}, 6 \mathrm{H}, 3 \times \mathrm{CH}_{2}\right) \mathrm{ppm}$ $\left.{ }^{13} \mathrm{C} \mathrm{NMR} \mathrm{(100} \mathrm{MHz}, \mathrm{CDCl}_{3}\right): \delta=146.5\left(\mathrm{C}_{\text {quart. }}, \mathrm{CArNH}_{2}\right), 136.2\left(+, 2 \times \mathrm{CH}_{\mathrm{Ar}}\right)$, 121.5 ( $\left.\mathrm{C}_{\text {quart. }}, \mathrm{CArS}_{\mathrm{Ar}}\right), 115.5\left(+, 2 \times \mathrm{CH}_{\mathrm{Ar}}\right), 53.7\left(-, 3 \times \mathrm{CH}_{2}\right), 46.4$ (C $\mathrm{C}_{\text {quart. }}$, $\mathrm{CArSC}_{\text {Ar }}, 28.4(+, \mathrm{CH}) \mathrm{ppm}$; IR (ATR): $\tilde{v}=3431(\mathrm{w}), 3342(\mathrm{~m}), 3213(\mathrm{w})$, $3021(v w), 2971(m), 2905(w), 2872(w), 1884(v w), 1631(m), 1593(m)$, $1491(\mathrm{~m}), 1423(\mathrm{w}), 1296(\mathrm{~m}), 1201(\mathrm{~m}), 1177(\mathrm{~m}), 1125(\mathrm{~m}), 1096(\mathrm{~m})$, $1063(\mathrm{w}), 1006(\mathrm{vw}), 935(\mathrm{vw}), 890(\mathrm{~m}), 814(\mathrm{~s}), 771(\mathrm{w}), 646(\mathrm{w}), 546(\mathrm{w})$, $517(\mathrm{~s}), 423(\mathrm{w}), 406(\mathrm{w}) \mathrm{cm}^{-1}$; MS (El, $\left.70 \mathrm{eV}\right): \mathrm{m} / \mathrm{z}(\%)=192(14)[\mathrm{M}+\mathrm{H}]^{+}$, $191(100)[\mathrm{M}]^{+}, 150(22)\left[\mathrm{M}-\mathrm{C}_{3} \mathrm{H}_{5}\right]^{+}, 125(65)\left[\mathrm{M}-\mathrm{C}_{5} \mathrm{H}_{7}+\mathrm{H}\right]^{+}, 124(62)[\mathrm{M}-$ $\left.\mathrm{C}_{5} \mathrm{H}_{7}\right]^{+}, 106$ (49) $\left[\mathrm{M}-\mathrm{C}_{5} \mathrm{H}_{8}-\mathrm{NH}_{2}\right]^{+} ; \mathrm{HRMS}(\mathrm{El}, 70 \mathrm{eV})$ : calcd for $\mathrm{C}_{11} \mathrm{H}_{13} \mathrm{~N}^{32} \mathrm{~S}$ $[\mathrm{M}]^{+}$191.0763; found 191.0764

Bicyclo[1.1.1]pent-1-yl(4-nitrophenyl)sulfane (7p): $7 p$ was synthesized from a solution of 1 (general procedure $b$ ) according to the general procedure $c$, but $6 p$ was added as a $0.5 \mathrm{M}$ solution in THF. The product $7 \mathrm{p}$ was obtained as a yellow solid that melts around room temperature in $47 \%$ yield $(62.0 \mathrm{mg}, 280 \mu \mathrm{mol})$. 
${ }^{1} \mathrm{H}$ NMR $\left(400 \mathrm{MHz}, \mathrm{CDCl}_{3}\right): \delta=8.15-8.12(\mathrm{~m}, 2 \mathrm{H}, \mathrm{Ar}-\mathrm{H}), 7.53-7.49(\mathrm{~m}$, $2 \mathrm{H}, \mathrm{Ar}-\mathrm{H}), 2.84(\mathrm{~s}, 1 \mathrm{H}, \mathrm{CH}), 2.13\left(\mathrm{~s}, 6 \mathrm{H}, 3 \times \mathrm{CH}_{2}\right) \mathrm{ppm} ;{ }^{13} \mathrm{C} \mathrm{NMR}(100 \mathrm{MHz}$ $\left.\mathrm{CDCl}_{3}\right): \delta=146.2\left(\mathrm{C}_{\text {quart. }}, \mathrm{C}_{\mathrm{Ar}} \mathrm{NO}_{2}\right), 145.6$ (C quart., $\left.C_{\mathrm{Ar}} \mathrm{S}\right), 130.7\left(+, 2 \times \mathrm{CH}_{\mathrm{Ar}}\right.$, $123.9\left(+, 2 \times \mathrm{CH}_{\mathrm{Ar}}\right), 54.7\left(-, 3 \times \mathrm{CH}_{2}\right), 44.6\left(\mathrm{C}_{\text {quart. }}, \mathrm{C}_{\mathrm{Ar}} \mathrm{SC}\right), 30.0(+, \mathrm{CH})$ ppm; IR (ATR): $\tilde{v}=2983$ (w), $2913(\mathrm{w}), 2877(\mathrm{w}), 1594(\mathrm{w}), 1575(\mathrm{~m})$, $1475(w), 1336(\mathrm{~s}), 1259(\mathrm{w}), 1208(\mathrm{~m}), 1124(\mathrm{~m}), 1090(\mathrm{~m}), 1012(\mathrm{w})$, $892(w), 851(\mathrm{~m}), 742(\mathrm{~m}), 684(\mathrm{w}), 535(\mathrm{w}), 408(\mathrm{vw}) \mathrm{cm}^{-1} ; \mathrm{MS}(\mathrm{EI}$, $70 \mathrm{eV}): m / z(\%)=222(6)[\mathrm{M}+1]^{+}, 221(38)[\mathrm{M}]^{+}, 180(7)\left[\mathrm{M}-\mathrm{C}_{3} \mathrm{H}_{5}\right]^{+}, 134$ (31) $\left[\mathrm{M}-\mathrm{C}_{3} \mathrm{H}_{5}-\mathrm{NO}_{2}\right]^{+}, 85$ (12) $\left[\mathrm{M}-\mathrm{C}_{6} \mathrm{H}_{4} \mathrm{NO}_{2}-\mathrm{CH}_{3}\right]^{+}, 67(100)\left[\mathrm{C}_{5} \mathrm{H}_{7}\right]^{+} ; \mathrm{HRMS}$ (El, $70 \mathrm{eV}$ ) calcd for $\mathrm{C}_{11} \mathrm{H}_{11} \mathrm{O}_{2} \mathrm{~N}^{32} \mathrm{~S}$ [M] 221.0505 ; found 221.0506 .

2-(Bicyclo[1.1.1]pent-1-ylthio)benzoic acid (7q): 7q was synthesized from a solution of $\mathbf{1}$ (general procedure $b$ ) according to the general procedure $c$, but $6 \mathbf{q}$ was added as a solid. Afterwards the crude product was purified by column chromatography $\left(\mathrm{SiO}_{2}, \quad\right.$ cyclohexane/EtOAc/AcOH, $5 / 1 / 0.01$ ). The product $\mathbf{7 q}$ was obtained as a white solid in $30 \%$ yield (29.0 mg, $132 \mu \mathrm{mol})$.

$R_{f}\left(\mathrm{SiO}_{2}\right.$, cyclohexane/EtOAc/AcOH, 5/1/0.01): 0.22; m.p. 125-127 ${ }^{\circ} \mathrm{C}$; ${ }^{1} \mathrm{H} \mathrm{NMR}\left(400 \mathrm{MHz}, \mathrm{CDCl}_{3}\right): \delta=8.17\left(\mathrm{dd}^{3}{ }^{3} \mathrm{~J}=7.8 \mathrm{~Hz},{ }^{4} \mathrm{~J}=1.5 \mathrm{~Hz}, 1 \mathrm{H}, \mathrm{Ar}-\right.$ H), $7.64\left(\mathrm{dd},{ }^{3} \mathrm{~J}=7.8 \mathrm{~Hz},{ }^{4} \mathrm{~J}=1.1 \mathrm{~Hz}, 1 \mathrm{H}, \operatorname{Ar}-\mathrm{H}\right), 7.50$ (ddd, ${ }^{3} \mathrm{~J}=7.8 \mathrm{~Hz}$, ${ }^{3} J=7.6 \mathrm{~Hz},{ }^{4} J=1.5 \mathrm{~Hz}, 1 \mathrm{H}, \mathrm{Ar}-\mathrm{H}$ ), 7.34 (ddd, ${ }^{3} J=7.8 \mathrm{~Hz},{ }^{3} J=7.6 \mathrm{~Hz}$, $\left.{ }^{3} J=1.1 \mathrm{~Hz}, 1 \mathrm{H}, \operatorname{Ar}-\mathrm{H}\right), 2.81(\mathrm{~s}, 1 \mathrm{H}, \mathrm{CH}), 2.08\left(\mathrm{~s}, 6 \mathrm{H}, 3 \times \mathrm{CH}_{2}\right) \mathrm{ppm}$ ${ }^{13} \mathrm{C} \mathrm{NMR}\left(100 \mathrm{MHz}, \mathrm{CDCl}_{3}\right): \delta=169.7$ (Cquart., $\left.\mathrm{CO}_{2} \mathrm{H}\right), 132.9$ (Cquart., $\mathrm{CArS}_{\mathrm{Ar}}$ ), $132.8\left(+, 2 \times \mathrm{CH}_{\mathrm{Ar}}\right), 132.7\left(+, \mathrm{CH}_{\mathrm{Ar}}\right), 129.9$ (Cquart., $\left.\mathrm{C}_{\mathrm{Ar}} \mathrm{CO}_{2} \mathrm{H}\right), 127.0(+$, $\mathrm{CH}_{\text {Ar }}$ ), $54.4\left(-, 3 \times \mathrm{CH}_{2}\right), 45.3$ (Cquart., $\mathrm{CArSC}_{\text {Ar }}, 29.8(+, \mathrm{CH}) \mathrm{ppm}$; IR (ATR) $\tilde{v}=2971(\mathrm{~m}), 2915(\mathrm{~m}), 2879(\mathrm{~m}), 2643(\mathrm{w}), 1673(\mathrm{~s}), 1587(\mathrm{w}), 1560(\mathrm{~m})$, $1463(\mathrm{~m}), 1434(\mathrm{w}), 1406(\mathrm{~m}), 1309(\mathrm{~m}), 1251(\mathrm{~s}), 1206(\mathrm{~m}), 1135(\mathrm{~m})$ $1056(\mathrm{~m}), 1043(\mathrm{~m}), 917(\mathrm{~m}), 893(\mathrm{~m}), 806(\mathrm{w}), 737(\mathrm{~s}), 692(\mathrm{~m}), 650(\mathrm{~m})$, $557(\mathrm{~m}), 491(\mathrm{w}), 464(\mathrm{w}), 416(\mathrm{w}) \mathrm{cm}^{-1} ; \mathrm{MS}(\mathrm{El}, 70 \mathrm{eV}): \mathrm{m} / \mathrm{z}(\%)=221(1)$ $[\mathrm{M}+1]^{+}, 220(4)[\mathrm{M}]^{+}, 179(10)\left[\mathrm{M}-\mathrm{C}_{3} \mathrm{H}_{5}\right]^{+}, 136(100)\left[\mathrm{M}-\mathrm{C}_{3} \mathrm{H}_{5}-\mathrm{CO}_{2}+\mathrm{H}\right]^{+}$, 109 (11) [M- $\left.\mathrm{C}_{5} \mathrm{H}_{7}-\mathrm{CO}_{2}\right]^{+}, 99(64)\left[\mathrm{M}-\mathrm{C}_{6} \mathrm{H}_{4} \mathrm{CO}_{2} \mathrm{H}\right]^{+}, 67(37)\left[\mathrm{C}_{5} \mathrm{H}_{7}\right]^{+} ; \mathrm{HRMS}$ (El, $70 \mathrm{eV}$ ): calcd for $\mathrm{C}_{12} \mathrm{H}_{12} \mathrm{O}_{2}{ }^{32} \mathrm{~S}[\mathrm{M}]^{+} 220.0558$; found 220.0559 .

For crystallographic data of this compound please use the supporting information. CCDC 1575329 contains the supplementary crystallographic data for this compound.

3-(Bicyclo[1.1.1]pent-1-ylthio)benzoic acid (7r): $7 \mathrm{r}$ was synthesized from a solution of 1 (general procedure a) according to the general procedure $\mathrm{c}$, but $6 r$ was added as a solid. Afterwards the crude product was purified by column chromatography $\left(\mathrm{SiO}_{2}\right.$, cyclohexane/EtOAc/AcOH, 5/1/0.01). The product $7 \mathrm{r}$ was obtained as a white solid in $53 \%$ yield $(63.0 \mathrm{mg}, 286 \mu \mathrm{mol})$.

$R_{f}\left(\mathrm{SiO}_{2}\right.$, cyclohexane/EtOAc/AcOH, 5/1/0.01): 0.29; m.p. $108-110^{\circ} \mathrm{C}$; ${ }^{1} \mathrm{H}$ NMR $\left(400 \mathrm{MHz}, \mathrm{CDCl}_{3}\right): \delta=8.12\left(\mathrm{dd}^{4}{ }^{4} \mathrm{~J}=1.8 \mathrm{~Hz},{ }^{4} \mathrm{~J}=1.8 \mathrm{~Hz}, 1 \mathrm{H}, \mathrm{Ar}-\right.$ H), 7.94 (ddd, ${ }^{3} J=7.8 \mathrm{~Hz},{ }^{4} \mathrm{~J}=1.8 \mathrm{~Hz},{ }^{4} \mathrm{~J}=1.3 \mathrm{~Hz}, 1 \mathrm{H}, \mathrm{Ar}-\mathrm{H}$ ), 7.60 (ddd, $\left.{ }^{3} J=7.7 \mathrm{~Hz},{ }^{4} J=1.8 \mathrm{~Hz},{ }^{4} J=1.3 \mathrm{~Hz}, 1 \mathrm{H}, \operatorname{Ar}-\mathrm{H}\right), 7.35\left(\mathrm{dd},{ }^{3} J=7.8 \mathrm{~Hz}\right.$ $\left.{ }^{3} J=7.7 \mathrm{~Hz}, 1 \mathrm{H}, \mathrm{Ar}-\mathrm{H}\right), 2.69(\mathrm{~s}, 1 \mathrm{H}, \mathrm{CH}), 1.93\left(\mathrm{~s}, 6 \mathrm{H}, 3 \times \mathrm{CH}_{2}\right) \mathrm{ppm}$; ${ }^{13} \mathrm{C}$ NMR $\left(100 \mathrm{MHz}, \mathrm{CDCl}_{3}\right): \delta=170.5\left(\mathrm{C}_{\text {quart. }}, \mathrm{CO}_{2} \mathrm{H}\right), 138.4\left(+, \mathrm{CH}_{\mathrm{Ar}}\right)$, 135.5 (Cquart., $\left.C_{\mathrm{Ar}} \mathrm{S}\right), 134.7\left(+, \mathrm{CH}_{\mathrm{Ar}}\right), 129.9\left(\mathrm{C}_{\text {quart. }} \mathrm{C}_{\mathrm{Ar}} \mathrm{CO}_{2} \mathrm{H}\right), 129.1(+$, $\left.\mathrm{CH}_{\text {Ar }}\right), 129.0\left(+, \mathrm{CH}_{\text {Ar }}\right), 54.3\left(-, 3 \times \mathrm{CH}_{2}\right), 45.5\left(\mathrm{C}_{\text {quart. }}, \mathrm{C}_{\mathrm{Ar}} \mathrm{SC}\right), 29.1(+, \mathrm{CH})$ ppm; IR (ATR): $\tilde{v}=2986(\mathrm{w}), 2906(\mathrm{w}), 2873(\mathrm{w}), 2544(\mathrm{w}), 1686(\mathrm{~s})$, $1591(w), 1570(w), 1474(w), 1420(m), 1290(m), 1260(m), 1204(m)$, $1165(\mathrm{w}), 1125(\mathrm{~m}), 1071(\mathrm{~m}), 928(\mathrm{~m}), 905(\mathrm{~m}), 886(\mathrm{~m}), 848(\mathrm{w}), 813(\mathrm{w})$ $746(\mathrm{~m}), 720(\mathrm{~m}), 677(\mathrm{~m}), 656(\mathrm{~m}), 548(\mathrm{~m}), 515(\mathrm{w}), 414(\mathrm{w}) \mathrm{cm}^{-1}$; MS (EI $70 \mathrm{eV}): m / z(\%)=221(5)[\mathrm{M}+1]^{+}, 220(36)\left[\mathrm{M}^{+}, 179(15)\left[\mathrm{M}-\mathrm{C}_{3} \mathrm{H}_{5}\right]^{+}, 154\right.$ (35) $\left[\mathrm{M}-\mathrm{C}_{5} \mathrm{H}_{7}+\mathrm{H}\right]^{+}, 135(28)\left[\mathrm{M}-\mathrm{C}_{3} \mathrm{H}_{5}-\mathrm{CO}_{2}\right]^{+}, 109$ (10) $\left[\mathrm{M}-\mathrm{C}_{5} \mathrm{H}_{7}-\mathrm{CO}_{2}\right]^{+}, 85$ (20) $\left[\mathrm{M}-\mathrm{CO}_{2} \mathrm{H}-\mathrm{C}_{6} \mathrm{H}_{5}-\mathrm{CH}_{2}\right]^{+}, 67(100)\left[\mathrm{C}_{5} \mathrm{H}_{7}\right]^{+} ; \mathrm{HRMS}(\mathrm{EI}, 70 \mathrm{eV})$ : calcd for $\mathrm{C}_{12} \mathrm{H}_{12} \mathrm{O}_{2}{ }^{32} \mathrm{~S}[\mathrm{M}]^{+} 220.0558$; found 220.0558 .
For crystallographic data of this compound please use the supporting information. CCDC 1575330 contains the supplementary crystallographic data for this compound.

Di(bicyclo[1.1.1]pent-1-yl)sulfane (11): In a quartz cuvette under argon atmosphere a $0.8 \mathrm{M}$ solution of $\mathrm{H}_{2} \mathrm{~S}$ in THF $(1.06 \mathrm{~mL}, 850 \mu \mathrm{mol}$, 1.00 equiv.) was added to a $0.37 \mathrm{M}$ solution of 1 (general procedure a) $(2.30 \mathrm{~mL}, 850 \mu \mathrm{mol}, 1.00$ equiv.) and irradiated for $1 \mathrm{~h}$ with a $4 \mathrm{~W}$ UV lamp $(254 \mathrm{~nm})$. The reaction mixture was diluted with $2 \mathrm{~mL} n$-pentane and washed with $1 \mathrm{M} \mathrm{NaOH}$ solution. The organic phase was dried over $\mathrm{Na}_{2} \mathrm{SO}_{4}$ and the solvent was removed under reduced pressure at room temperature to obtain the product 11 as a volatile, pale yellow liquid in $64 \%$ yield $(90.0 \mathrm{mg}, 540 \mu \mathrm{mol})$.

${ }^{1} \mathrm{H} \mathrm{NMR}\left(400 \mathrm{MHz}, \mathrm{CDCl}_{3}\right): \delta=2.71(\mathrm{~s}, 2 \mathrm{H}, 2 \times \mathrm{CH}), 2.04(\mathrm{~s}, 12 \mathrm{H}$ $\left.6 \times \mathrm{CH}_{2}\right) \mathrm{ppm} ;{ }^{13} \mathrm{C} \mathrm{NMR}\left(100 \mathrm{MHz}, \mathrm{CDCl}_{3}\right): \delta=55.2\left(-, 6 \times \mathrm{CH}_{2}\right), 44.2$ (Cquart., $2 \times \mathrm{SC}), 30.1(+, 2 \times \mathrm{CH}) \mathrm{ppm}$; IR (ATR): $\tilde{v}=2975(\mathrm{~m}), 2909(\mathrm{~m})$, $2874(\mathrm{~m}), 1447(\mathrm{vw}), 1201(\mathrm{~s}), 1131(\mathrm{~m}), 894(\mathrm{~m}), 471(\mathrm{w}) \mathrm{cm}^{-1}$; MS (EI, $70 \mathrm{eV}): m / z(\%)=166(9)[\mathrm{M}]^{+}, 125(16)\left[\mathrm{M}-\mathrm{C}_{3} \mathrm{H}_{5}\right]^{+}, 99(34)\left[\mathrm{M}-\mathrm{C}_{5} \mathrm{H}_{7}\right]^{+}, 85$ (54) $\left[\mathrm{M}-\left(\mathrm{C}_{3} \mathrm{H}_{5}\right)_{2}+\mathrm{H}\right]^{+}, 67(100)\left[\mathrm{C}_{5} \mathrm{H}_{7}\right]^{+}$; HRMS (El, $\left.70 \mathrm{eV}\right)$ : calcd for $\mathrm{C}_{10} \mathrm{H}_{14}{ }^{32} \mathrm{~S}[\mathrm{M}]^{+}$166.0811; found 166.0812 .

Bicyclo[1.1.1]pent-1-yl(propyl)sulfane (9a): 9a was synthesized from a solution of 1 (general procedure a) according to the general procedure $c$. The product $9 \mathrm{a}$ was obtained as a volatile, pale yellow liquid in $81 \%$ yield (42.0 mg, $295 \mu \mathrm{mol}$ ).

${ }^{1} \mathrm{H} \mathrm{NMR}\left(400 \mathrm{MHz}, \mathrm{CDCl}_{3}\right): \delta=2.72(\mathrm{~s}, 1 \mathrm{H}, \mathrm{CH}), 2.51\left(\mathrm{t},{ }^{3} \mathrm{~J}=7.3 \mathrm{~Hz}, 2 \mathrm{H}\right.$, $\left.\mathrm{CH}_{2} \mathrm{CH}_{2} \mathrm{CH}_{3}\right), 1.96\left(\mathrm{~s}, 6 \mathrm{H}, 3 \times \mathrm{CCH}_{2} \mathrm{CH}\right.$ ), 1.60 (td, ${ }^{3} \mathrm{~J}=7.4 \mathrm{~Hz},{ }^{3} \mathrm{~J}=7.3 \mathrm{~Hz}$, $\left.2 \mathrm{H}, \mathrm{CH}_{2} \mathrm{CH}_{2} \mathrm{CH}_{3}\right), 0.98$ (t, $\left.{ }^{3} \mathrm{~J}=7.4 \mathrm{~Hz}, 3 \mathrm{H}, \mathrm{CH}_{3}\right) \mathrm{ppm} ;{ }^{13} \mathrm{C} \mathrm{NMR}(100 \mathrm{MHz}$, $\left.\mathrm{CDCl}_{3}\right): \delta=53.9\left(-, 3 \times \mathrm{CCH}_{2} \mathrm{CH}\right), 44.7\left(\mathrm{C}_{\text {quart. }}, \mathrm{CCH}_{2} \mathrm{CH}\right), 33.3(-$, $\left.\mathrm{CH}_{2} \mathrm{CH}_{2} \mathrm{CH}_{3}\right), 28.8(+, \mathrm{CH}), 23.9\left(-, \mathrm{CH}_{2} \mathrm{CH}_{2} \mathrm{CH}_{3}\right), 13.7\left(+, \mathrm{CH}_{3}\right)$ ppm; IR (ATR): $\tilde{v}=2961(\mathrm{~m}), 2907(\mathrm{w}), 2872(\mathrm{w}), 1450(\mathrm{w}), 1376(\mathrm{vw}), 1291(\mathrm{vw})$, $1205(\mathrm{~m}), 1140(\mathrm{~m}), 902(\mathrm{w}), 797(\mathrm{vw}) \mathrm{cm}^{-1}$. Due to the nonpolar and volatile nature of this compound no HRMS measurement was possible with $\mathrm{EI}$ or FAB. In the ESI (S10) we attached a GC-MS spectrum of 9a.

Bicyclo[1.1.1]pent-1-yl(isopropyl)sulfane (9b): $9 b$ was synthesized from a solution of $\mathbf{1}$ (general procedure a) according to the general procedure $\mathrm{c}$. The product $\mathbf{9 b}$ was obtained as a volatile, colourless liquid in $35 \%$ yield (18.0 mg, $127 \mu \mathrm{mol})$.

${ }^{1} \mathrm{H}$ NMR $\left(400 \mathrm{MHz}, \mathrm{CDCl}_{3}\right): \delta=2.96$ (sept, $\left.{ }^{3} J=6.8 \mathrm{~Hz}, 1 \mathrm{H}, \mathrm{CH}\left(\mathrm{CH}_{3}\right)_{2}\right)$ $2.71\left(\mathrm{~s}, 1 \mathrm{H}, \mathrm{CH}\left(\mathrm{CH}_{2}\right)_{3}\right), 2.00\left(\mathrm{~s}, 6 \mathrm{H}, 3 \times \mathrm{CH}_{2}\right), 1.28\left(\mathrm{~d},{ }^{3} \mathrm{~J}=6.8 \mathrm{~Hz}, 6 \mathrm{H}\right.$, $\left.2 \times \mathrm{CH}_{3}\right) \mathrm{ppm} ;{ }^{13} \mathrm{C}$ NMR $\left(100 \mathrm{MHz}, \mathrm{CDCl}_{3}\right): \delta=54.6\left(-, 3 \times \mathrm{CH}_{2}\right), 44.5$ (C quart., $\mathrm{CHSC}), 35.8\left(+, \mathrm{CH}\left(\mathrm{CH}_{3}\right)_{2}\right), 29.2\left(+, \mathrm{CH}\left(\mathrm{CH}_{2}\right)_{3}\right), 24.8\left(+, 2 \times \mathrm{CH}_{3}\right)$ ppm; IR (ATR): $\tilde{v}=2974(\mathrm{w}), 2908(\mathrm{w}), 2873(\mathrm{w}), 1447(\mathrm{vw}), 1381(\mathrm{vw})$, $1364(\mathrm{vw}), \quad 1243(\mathrm{vw}), \quad 1206(\mathrm{~m}), \quad 1139(\mathrm{w}), \quad 1050(\mathrm{w}), \quad 903(\mathrm{vw})$ $646(\mathrm{vw}) \mathrm{cm}^{-1}$. Due to the nonpolar and volatile nature of this compound no HRMS measurement was possible with El or FAB. In the ESI (S11) we attached a GC-MS spectrum of $\mathbf{9 b}$.

Bicyclo[1.1.1]pent-1-yl(butyl)sulfane (9c): 9c was synthesized from a solution of 1 (general procedure a) according to the general procedure $c$. The product $9 \mathrm{c}$ was obtained as a volatile, pale yellow liquid in $67 \%$ yield $(38.0 \mathrm{mg}, 243 \mu \mathrm{mol})$.

${ }^{1} \mathrm{H} \mathrm{NMR}\left(400 \mathrm{MHz}, \mathrm{CDCl}_{3}\right): \delta=2.72(\mathrm{~s}, 1 \mathrm{H}, \mathrm{CH}), 2.53\left(\mathrm{t},{ }^{3} \mathrm{~J}=7.4 \mathrm{~Hz}, 2 \mathrm{H}\right.$, $\left.\mathrm{CH}_{2} \mathrm{CH}_{2} \mathrm{CH}_{2} \mathrm{CH}_{3}\right), 1.96\left(\mathrm{~s}, 6 \mathrm{H}, 3 \times \mathrm{CCH}_{2} \mathrm{CH}\right), 1.61-1.52(\mathrm{~m}, 2 \mathrm{H}$, $\mathrm{CH}_{2} \mathrm{CH}_{2} \mathrm{CH}_{2} \mathrm{CH}_{3}$ ), 1.44-1.35 (m, 2H, $\left.\mathrm{CH}_{2} \mathrm{CH}_{2} \mathrm{CH}_{2} \mathrm{CH}_{3}\right), 0.91$ (t, ${ }^{3} \mathrm{~J}=7.3 \mathrm{~Hz}$, $\left.3 \mathrm{H}, \mathrm{CH}_{3}\right) \mathrm{ppm} ;{ }^{13} \mathrm{C} \mathrm{NMR}\left(100 \mathrm{MHz}, \mathrm{CDCl}_{3}\right): \delta=53.9\left(-, 3 \times \mathrm{CCH}_{2} \mathrm{CH}\right)$, 44.7 (Cquart., $\left.\quad \mathrm{CCH}_{2} \mathrm{CH}\right), \quad 33.3 \quad\left(-, \quad \mathrm{CH}_{2} \mathrm{CH}_{2} \mathrm{CH}_{2} \mathrm{CH}_{3}\right), \quad 31.0 \quad$ (-, $\left.\mathrm{CH}_{2} \mathrm{CH}_{2} \mathrm{CH}_{2} \mathrm{CH}_{3}\right), 28.8(+, \mathrm{CH}), 22.2\left(-, \mathrm{CH}_{2} \mathrm{CH}_{2} \mathrm{CH}_{2} \mathrm{CH}_{3}\right), 13.9\left(+, \mathrm{CH}_{3}\right)$ 
ppm; IR (ATR): $\tilde{v}=2959(\mathrm{w}), 2907(\mathrm{w}), 2872(\mathrm{w}), 1458(\mathrm{vw}), 1377(\mathrm{vw})$, $1274(\mathrm{vw}), 1205(\mathrm{~m}), 1141(\mathrm{w}), 903(\mathrm{w}), 745(\mathrm{vw}), 395(\mathrm{vw}) \mathrm{cm}^{-1}$. Due to the nonpolar and volatile nature of this compound no HRMS measurement was possible with EI or FAB. In the ESI (S12) we attached a GC-MS spectrum of $9 c$.

Bicyclo[1.1.1]pent-1-yl(tert-butyl)sulfane (9d): $9 \mathrm{~d}$ was synthesized from a solution of 1 (general procedure a) according to the general procedure $\mathrm{c}$. The product $9 \mathbf{d}$ was obtained as a volatile, pale yellow liquid in $39 \%$ yield (22.0 mg, $141 \mu \mathrm{mol}$ ).

${ }^{1} \mathrm{H} \mathrm{NMR}\left(400 \mathrm{MHz}, \mathrm{CDCl}_{3}\right): \delta=2.69(\mathrm{~s}, 1 \mathrm{H}, \mathrm{CH}), 2.08\left(\mathrm{~s}, 6 \mathrm{H}, 3 \times \mathrm{CH}_{2}\right)$, $1.36\left(\mathrm{~s}, 9 \mathrm{H}, 3 \times \mathrm{CH}_{3}\right) \mathrm{ppm} ;{ }^{13} \mathrm{C} \mathrm{NMR}\left(100 \mathrm{MHz}, \mathrm{CDCl}_{3}\right): \delta=56.1(-$, $\left.3 \times \mathrm{CH}_{2}\right), 44.8$ ( $\left.\mathrm{C}_{\text {quart. }}, \quad \mathrm{CSC}\left(\mathrm{CH}_{3}\right)_{3}\right), 44.1$ ( $\left.\mathrm{C}_{\text {quart. }}, \mathrm{C}\left(\mathrm{CH}_{3}\right)_{3}\right), 32.0(+$, $\left.3 \times \mathrm{CH}_{3}\right), 30.7(+, \mathrm{CH}) \mathrm{ppm}$; IR (ATR): $\tilde{v}=2962(\mathrm{w}), 2910(\mathrm{w}), 2874(\mathrm{w})$, $1456(w), 1362(w), 1260(v w), 1208(m), 1164(w), 1135(w), 903(w)$ $803(\mathrm{vw}), 597(\mathrm{vw}) \mathrm{cm}^{-1}$. Due to the nonpolar and volatile nature of this compound no HRMS measurement was possible with EI or FAB. In the ESI (S13) we attached a GC-MS spectrum of $\mathbf{9 d}$.

2-(Bicyclo[1.1.1]pent-1-ylthio)ethan-1-ol (9e): 9e was synthesized from a solution of 1 (general procedure a) according to the general procedure $\mathrm{c}$. The organic phase was not washed with $\mathrm{NaOH}$ solution, but concentrated and purified by column chromatography $\left(\mathrm{SiO}_{2}\right.$, cyclohexane/EtOAc, $\left.2: 1\right)$ The product $9 \mathrm{e}$ was obtained as a colourless oil in $77 \%$ yield $(88.0 \mathrm{mg}$, $610 \mu \mathrm{mol})$.

$R_{\mathrm{f}}\left(\mathrm{SiO}_{2}\right.$, cyclohexane/EtOAc, $\left.2: 1\right): 0.44 ;{ }^{1} \mathrm{H} \mathrm{NMR}\left(400 \mathrm{MHz}, \mathrm{CDCl}_{3}\right): \delta=$ $3.70\left(\mathrm{td},{ }^{3} \mathrm{~J}=6.2 \mathrm{~Hz},{ }^{3} \mathrm{~J}=6.1 \mathrm{~Hz}, 2 \mathrm{H}, \mathrm{CH}_{2} \mathrm{CH}_{2} \mathrm{OH}\right), 2.75\left(\mathrm{t},{ }^{3} \mathrm{~J}=6.1 \mathrm{~Hz}, 2 \mathrm{H}\right.$, $\left.\mathrm{CH}_{2} \mathrm{CH}_{2} \mathrm{OH}\right), 2.73(\mathrm{~s}, 1 \mathrm{H}, \mathrm{CH}), 2.09\left(\mathrm{t},{ }^{3} \mathrm{~J}=6.2 \mathrm{~Hz}, 1 \mathrm{H}, \mathrm{OH}\right), 1.98(\mathrm{~s}, 6 \mathrm{H}$, $\left.3 \times \mathrm{CCH}_{2} \mathrm{CH}\right) \mathrm{ppm} ;{ }^{13} \mathrm{C} \mathrm{NMR}\left(100 \mathrm{MHz}, \mathrm{CDCl}_{3}\right): \delta=61.4\left(-, \mathrm{CH}_{2} \mathrm{CH}_{2} \mathrm{OH}\right)$, $54.1\left(-, 3 \times \mathrm{CCH}_{2} \mathrm{CH}\right), 44.2$ (Cquart., $\left.\mathrm{CS}\right), 34.6\left(-, \mathrm{CH}_{2} \mathrm{CH}_{2} \mathrm{OH}\right), 28.8(+, \mathrm{CH})$ ppm; IR (ATR): $\tilde{v}=3335(\mathrm{w}), 2974(\mathrm{~m}), 2907(\mathrm{w}), 2872(\mathrm{~m}), 1407(\mathrm{w})$, $1287(\mathrm{vw}), 1206(\mathrm{~m}), 1137(\mathrm{~m}), 1041(\mathrm{~m}), 1008(\mathrm{~m}), 929(\mathrm{w}), 902(\mathrm{w})$, $763(\mathrm{w}), 667(\mathrm{w}), 440(\mathrm{vw}) \mathrm{cm}^{-1}$; MS (El, $\left.70 \mathrm{eV}\right): \mathrm{m} / \mathrm{z}(\%)=145(1)[\mathrm{M}+\mathrm{H}]^{+}$, $143(1)[\mathrm{M}-\mathrm{H}]^{+}, 100(70)\left[\mathrm{M}-\mathrm{C}_{2} \mathrm{H}_{4} \mathrm{OH}+\mathrm{H}\right]^{+}, 99(37)\left[\mathrm{M}-\mathrm{C}_{2} \mathrm{H}_{4} \mathrm{OH}\right]^{+}, 85(100)$ $\left[\mathrm{M}-\mathrm{C}_{2} \mathrm{H}_{4} \mathrm{OH}-\mathrm{CH}_{2}\right]^{+}, 77(10)\left[\mathrm{M}-\mathrm{C}_{5} \mathrm{H}_{7}\right]^{+}, 67(64)\left[\mathrm{C}_{5} \mathrm{H}_{7}\right]^{+}$; $\mathrm{HRMS}(\mathrm{El}, 70 \mathrm{eV})$ calcd for $\mathrm{C}_{7} \mathrm{H}_{12} \mathrm{O}_{1}{ }^{32} \mathrm{~S}_{1}[\mathrm{M}]^{+}$144.0603; found 144.0605 .

2-(Bicyclo[1.1.1]pent-1-ylthio)acetic acid (9f): 9f was synthesized from a solution of 1 (general procedure a) according to the general procedure $c$. The organic phase was not washed with $\mathrm{NaOH}$ solution, but concentrated and purified by column chromatography $\left(\mathrm{SiO}_{2}\right.$, cyclohexane/EtOAc/ trifluoroacetic acid, 10:1:0.01). The product 9f was obtained as a colourless oil in $66 \%$ yield $(59.0 \mathrm{mg}, 373 \mu \mathrm{mol})$.

$R_{f} \quad\left(\mathrm{SiO}_{2}, \quad\right.$ cyclohexane/EtOAc/trifluoroacetic acid, 10:1:0.01): 0.20 ${ }^{1} \mathrm{H}$ NMR $\left(400 \mathrm{MHz}, \mathrm{CDCl}_{3}\right): \delta=3.31\left(\mathrm{~s}, 2 \mathrm{H}, \mathrm{CSCH}_{2}\right), 2.75(\mathrm{~s}, 1 \mathrm{H}, \mathrm{CH})$, $2.01\left(\mathrm{~s}, 6 \mathrm{H}, 3 \times \mathrm{CCH}_{2} \mathrm{CH}\right) \mathrm{ppm} ;{ }^{13} \mathrm{C} \mathrm{NMR}\left(100 \mathrm{MHz}, \mathrm{CDCl}_{3}\right): \delta=175.3$ (Cquart., $\left.\mathrm{CO}_{2} \mathrm{H}\right), 53.6\left(-, 3 \times \mathrm{CCH}_{2} \mathrm{CH}\right), 44.3\left(\mathrm{C}_{\text {quart. }}, \mathrm{CSCH}_{2}\right), 33.2(-$, $\left.\mathrm{CSCH}_{2}\right), 28.7(+, \mathrm{CH}) \mathrm{ppm}$; IR (ATR): $\tilde{v}=2979(\mathrm{~m}), 2910(\mathrm{~m}), 2876(\mathrm{~m})$, $2670(\mathrm{w}), 1705(\mathrm{~s}), 1419(\mathrm{w}), 1291(\mathrm{~m}), 1206(\mathrm{~m}), 1134(\mathrm{~m}), 900(\mathrm{~m})$, 785 (w), 668 (w), 581 (w), $464(w) \mathrm{cm}^{-1}$; MS (El, $\left.70 \mathrm{eV}\right): \mathrm{m} / \mathrm{z}(\%)=158(1)$ $[\mathrm{M}]^{+}, 157(2)[\mathrm{M}-\mathrm{H}]^{+}, 99(100)\left[\mathrm{M}-\mathrm{CH}_{2} \mathrm{CO}_{2} \mathrm{H}\right]^{+}, 67(79)\left[\mathrm{C}_{5} \mathrm{H}_{7}\right]^{+} ; \mathrm{HRMS}(\mathrm{EI}$, $70 \mathrm{eV}$ ): calcd for $\mathrm{C}_{7} \mathrm{H}_{10} \mathrm{O}_{2}{ }^{32} \mathrm{~S}[\mathrm{M}]+{ }^{+}$158.0402; found 158.0403 .

Benzyl(bicyclo[1.1.1]pent-1-yl)sulfane (9g): $9 \mathrm{~g}$ was synthesized from a solution of 1 (general procedure a) according to the general procedure $c$. The product $9 \mathrm{~g}$ was obtained as a pale yellow oil in $53 \%$ yield $(94.0 \mathrm{mg}$, $494 \mu \mathrm{mol})$.

${ }^{1} \mathrm{H} \mathrm{NMR}\left(400 \mathrm{MHz}, \mathrm{CDCl}_{3}\right): \delta=7.32-7.17(\mathrm{~m}, 5 \mathrm{H}, \mathrm{Ar}-\mathrm{H}), 3.73(\mathrm{~s}, 2 \mathrm{H}$, $\left.\mathrm{C}_{6} \mathrm{H}_{5} \mathrm{CH}_{2} \mathrm{~S}\right), 2.64(\mathrm{~s}, 1 \mathrm{H}, \mathrm{CH}), 1.68\left(\mathrm{~s}, 6 \mathrm{H}, 3 \times \mathrm{CCH}_{2} \mathrm{CH}\right) \mathrm{ppm} ;{ }^{13} \mathrm{C}$ NMR $\left(100 \mathrm{MHz}, \mathrm{CDCl}_{3}\right): \delta=138.9\left(\mathrm{C}_{\text {quart. }}, \mathrm{C}_{\mathrm{Ar}}\right), 128.9\left(+, 2 \times \mathrm{CH}_{\mathrm{Ar}}\right), 128.5(+$, $\left.2 \times \mathrm{CH}_{\mathrm{Ar}}\right), 127.0\left(+, \mathrm{CH}_{\mathrm{Ar}}\right), 53.8\left(-, 3 \times \mathrm{CCH}_{2} \mathrm{CH}\right), 44.8$ ( $\left.\mathrm{C}_{\text {quart. }}, \mathrm{SC}\right), 35.9$ $\left(-, \mathrm{C}_{\mathrm{Ar}} \mathrm{CH}_{2} \mathrm{~S}\right), 29.0(+, \mathrm{CH})$ ppm; IR (ATR): $\tilde{v}=3060(\mathrm{vw}), 3026(\mathrm{vw})$, $2974(w), 2906(w), 2872(w), 1600(v w), 1493(w), 1451(w), 1205(m)$, $1137(\mathrm{w}), 1068(\mathrm{w}), 1028(\mathrm{vw}), 903(\mathrm{vw}), 862(\mathrm{vw}), 760(\mathrm{vw}), 696(\mathrm{~m})$, $563(\mathrm{vw}), 472(\mathrm{vw}), 440(\mathrm{vw}) \mathrm{cm}^{-1}$; MS (El, $\left.70 \mathrm{eV}\right): \mathrm{m} / \mathrm{z}(\%)=190(1)[\mathrm{M}]^{+}$, 99 (4) $\left[\mathrm{M}-\mathrm{C}_{6} \mathrm{H}_{5} \mathrm{CH}_{2}\right]^{+}, 91(100)\left[\mathrm{M}-\mathrm{C}_{5} \mathrm{H}_{7} \mathrm{~S}\right]^{+}, 67(3)\left[\mathrm{C}_{5} \mathrm{H}_{7}\right]^{+}$; HRMS (EI, $70 \mathrm{eV}) \mathrm{C}_{12} \mathrm{H}_{14}{ }^{32} \mathrm{~S}[\mathrm{M}]^{+} 190.0811$; found 190.0811

Fmoc-Bicyclo[1.1.1]pentyl-Cys-OtBu (9h): 9h was synthesized from a solution of 1 (general procedure a) according to the general procedure $c$, but $8 \mathrm{~h}$ (ESI S3) was added as a $1 \mathrm{M}$ solution in THF. Afterwards the crude product was purified by column chromatography $\left(\mathrm{SiO}_{2}\right.$ cyclohexane/EtOAc, 10:1). The product $\mathbf{9 h}$ was obtained as a colourless oil in $45 \%$ yield ( $172 \mathrm{mg}, 369 \mu \mathrm{mol})$.

$R_{f}\left(\mathrm{SiO}_{2}\right.$, cyclohexane/EtOAc, 10:1): 0.27; ${ }^{1} \mathrm{H} \mathrm{NMR}\left(400 \mathrm{MHz}, \mathrm{CDCl}_{3}\right)$ $\delta=7.78-7.75(\mathrm{~m}, 2 \mathrm{H}, \mathrm{Ar}-\mathrm{H}), 7.62-7.60(\mathrm{~m}, 2 \mathrm{H}, \mathrm{Ar}-\mathrm{H}), 7.42-7.38(\mathrm{~m}, 2 \mathrm{H}$, Ar-H), 7.34-7.29 (m, 2H, Ar-H), $5.56\left(\mathrm{~d},{ }^{3} \mathrm{~J}=7.8 \mathrm{~Hz}, 1 \mathrm{H}, \mathrm{NH}\right), 4.51(\mathrm{dt}$, $\left.{ }^{3} J=7.8 \mathrm{~Hz}, \quad{ }^{3} J=4.8 \mathrm{~Hz}, \quad 1 \mathrm{H}, \quad \mathrm{SCH}_{2} \mathrm{CH}\right), 4.41\left(\mathrm{~d},{ }^{3} J=7.0 \mathrm{~Hz}, \quad 2 \mathrm{H}\right.$, $\mathrm{CO}_{2} \mathrm{CH}_{2} \mathrm{CH}$ ), $4.23\left(\mathrm{t},{ }^{3} \mathrm{~J}=7.0 \mathrm{~Hz}, 1 \mathrm{H}, \mathrm{CO}_{2} \mathrm{CH}_{2} \mathrm{CH}\right.$ ), $3.02\left(\mathrm{dd},{ }^{2} \mathrm{~J}=13.6 \mathrm{~Hz}\right.$, $\left.{ }^{3} J=4.8 \mathrm{~Hz}, 1 \mathrm{H}, \quad \mathrm{SCH}_{2} \mathrm{CH}\right), 2.94\left(\mathrm{dd},{ }^{2} \mathrm{~J}=13.6 \mathrm{~Hz},{ }^{3} J=4.8 \mathrm{~Hz}, 1 \mathrm{H}\right.$ $\left.\mathrm{SCH}_{2} \mathrm{CH}\right), 2.70\left(\mathrm{~s}, 1 \mathrm{H}, \mathrm{SCCH}_{2} \mathrm{CH}\right), 1.93\left(\mathrm{~s}, 6 \mathrm{H}, 3 \times \mathrm{SCCH}_{2} \mathrm{CH}\right), 1.48(\mathrm{~s}$, $\left.9 \mathrm{H}, 3 \times \mathrm{CH}_{3}\right) \mathrm{ppm} ;{ }^{13} \mathrm{C} \mathrm{NMR}\left(100 \mathrm{MHz}, \mathrm{CDCl}_{3}\right): \delta=169.7$ (Cquart., $\left.\mathrm{CCO}_{2}\right)$ 155.8 (Cquart., $\mathrm{NCO}_{2}$ ), 144.0 (C $\mathrm{C}_{\text {quart. }}, \mathrm{C}_{\mathrm{Ar}}$ ), 143.9 (Cquart., $\mathrm{C}_{\mathrm{Ar}}$ ), 141.5 (Cquart., $\left.2 \times C_{\mathrm{Ar}}\right), 127.9\left(+, 2 \times \mathrm{CH}_{\mathrm{Ar}}\right), 127.2\left(+, 2 \times \mathrm{CH}_{\mathrm{Ar}}\right), 125.3\left(+, \mathrm{CH}_{\mathrm{Ar}}\right), 125.2(+$ $\left.\mathrm{CH}_{\text {Ar }}\right), 120.1\left(+, 2 \times \mathrm{CH}_{\mathrm{Ar}}\right), 82.9\left(\mathrm{C}_{\text {quart. }}, \mathrm{CCH}_{3}\right), 67.2\left(-, \mathrm{CO}_{2} \mathrm{CH}_{2} \mathrm{CH}\right), 54.4$ (+, $\left.\mathrm{SCH}_{2} \mathrm{CH}\right), 53.8(-, 3 \times \mathrm{SCCH} 2 \mathrm{CH}), 47.3\left(+, \mathrm{CO}_{2} \mathrm{CH}_{2} \mathrm{CH}\right), 44.4$ (C) quart. $\left.\mathrm{SCCH}_{2} \mathrm{CH}\right), 33.9\left(-, \mathrm{SCH}_{2} \mathrm{CH}\right), 28.6\left(+, \mathrm{SCCH}_{2} \mathrm{CH}\right), 28.2\left(+, 3 \times \mathrm{CH}_{3}\right)$ ppm; IR (ATR): $\tilde{v}=3331(\mathrm{vw}), 2976(\mathrm{w}), 2908(\mathrm{vw}), 2874(\mathrm{w}), 1712(\mathrm{~m})$ $1503(w), 1449(w), 1393(v w), 1368(w), 1342(w), 1207(m), 1151(m)$, $1105(w), 1047(w), 999(w), 901(v w), 844(w), 757(w), 738(m), 621(v w)$, $536(\mathrm{w}), 424(\mathrm{w}) \mathrm{cm}^{-1} ; \mathrm{MS}(\mathrm{El}, 70 \mathrm{eV}): \mathrm{m} / z(\%)=466(1)[\mathrm{M}+\mathrm{H}]^{+}, 465(2)$ $[\mathrm{M}]^{+}, 408(15)\left[\mathrm{M}-\mathrm{C}\left(\mathrm{CH}_{3}\right)_{3}\right]^{+}, 365(38)\left[\mathrm{M}-\mathrm{CO}_{2} \mathrm{C}\left(\mathrm{CH}_{3}\right)_{3}+\mathrm{H}\right]^{+}, 364(100)[\mathrm{M}-$ $\left.\mathrm{CO}_{2} \mathrm{C}\left(\mathrm{CH}_{3}\right)_{3}\right]^{+}, 179(65)\left[\mathrm{C}_{14} \mathrm{H}_{10}+\mathrm{H}\right]^{+}, 178(100)\left[\mathrm{C}_{14} \mathrm{H}_{10}\right]^{+}$; HRMS (El, $70 \mathrm{eV}$ ): calcd for $\mathrm{C}_{27} \mathrm{H}_{31} \mathrm{O}_{4} \mathrm{~N}^{32} \mathrm{~S}[\mathrm{M}]+465.1968$; found 465.1970 .

1,2-Bis(bicyclo[1.1.1]pent-1-ylthio)ethane (13a): 13a was synthesized from a solution of 1 (general procedure a) according to the general procedure $\mathrm{c}$. The product 13a was obtained as a white solid in $31 \%$ yield (82.0 mg, $362 \mu \mathrm{mol})$.

m.p. $65-67^{\circ} \mathrm{C} ;{ }^{1} \mathrm{H} \mathrm{NMR}\left(400 \mathrm{MHz}, \mathrm{CDCl}_{3}\right): \delta=2.74(\mathrm{~s}, 2 \mathrm{H}, 2 \times \mathrm{CH}), 2.72$ $\left(\mathrm{s}, 4 \mathrm{H}, 2 \times \mathrm{SCH}_{2}\right), 1.98\left(\mathrm{~s}, 12 \mathrm{H}, 6 \times \mathrm{CCH}_{2} \mathrm{CH}\right) \mathrm{ppm} ;{ }^{13} \mathrm{C} \mathrm{NMR}(100 \mathrm{MHz}$ $\left.\mathrm{CDCl}_{3}\right): \delta=54.1\left(-, 6 \times \mathrm{CCH}_{2} \mathrm{CH}\right), 44.5$ (Cquart., $\left.2 \times \mathrm{SC}\right), 32.2(-$, $\left.2 \times \mathrm{SCH}_{2}\right), 28.8(+, 2 \times \mathrm{CH}) \mathrm{ppm}$; IR (ATR): $\tilde{v}=2968(\mathrm{~s}), 2904(\mathrm{~m})$, $2869(\mathrm{~m}), 2345(\mathrm{vw}), 1734(\mathrm{vw}), 1448(\mathrm{w}), 1420(\mathrm{~m}), 1261(\mathrm{vw}), 1205(\mathrm{~s})$, $1135(\mathrm{~s}), 921(\mathrm{w}), 904(\mathrm{~m}), 884(\mathrm{w}), 801(\mathrm{w}), 726(\mathrm{~m}), 694(\mathrm{~m}), 542(\mathrm{w})$, 448 (w) $\mathrm{cm}^{-1} ; \mathrm{MS}(\mathrm{El}, 70 \mathrm{eV}): \mathrm{m} / \mathrm{z}(\%)=226(14)[\mathrm{M}]^{+}, 159(3)\left[\mathrm{M}-\mathrm{C}_{5} \mathrm{H}_{7}\right]^{+}$, $127(34)\left[\mathrm{M}-\mathrm{C}_{5} \mathrm{H}_{7} \mathrm{~S}\right]^{+}, 99(92)\left[\mathrm{M}-\mathrm{C}_{5} \mathrm{H}_{7} \mathrm{~S}-\mathrm{C}_{2} \mathrm{H}_{4}\right]^{+}, 98(90)\left[\mathrm{C}_{5} \mathrm{H}_{6} \mathrm{~S}\right]^{+}, 85$ (34) [M- $\left.\mathrm{C}_{5} \mathrm{H}_{7} \mathrm{~S}-\mathrm{C}_{2} \mathrm{H}_{4}-\mathrm{CH}_{2}\right]^{+}, 67(100)\left[\mathrm{C}_{5} \mathrm{H}_{7}\right]^{+}$; HRMS (EI, $\left.70 \mathrm{eV}\right)$ : calcd for $\mathrm{C}_{12} \mathrm{H}_{18}{ }^{32} \mathrm{~S}_{2}[\mathrm{M}]^{+} 226.0844$; found 226.0843 .

1,4-Bis(bicyclo[1.1.1]pent-1-ylthio)butane (13b): 13b was synthesized from a solution of $\mathbf{1}$ (general procedure a) according to the general procedure $\mathrm{c}$. The product $\mathbf{1 3 \mathbf { b }}$ was obtained as a colourless liquid in $31 \%$ yield $(65.0 \mathrm{mg}, 255 \mu \mathrm{mol})$.

${ }^{1} \mathrm{H} \mathrm{NMR}\left(400 \mathrm{MHz}, \mathrm{CDCl}_{3}\right): \delta=2.72(\mathrm{~s}, 2 \mathrm{H}, 2 \times \mathrm{CH}), 2.53(\mathrm{~m}, 4 \mathrm{H}$ $\left.2 \times \mathrm{SCH}_{2} \mathrm{CH}_{2}\right), 1.96\left(\mathrm{~s}, 12 \mathrm{H}, 6 \times \mathrm{CCH}_{2} \mathrm{CH}\right), 1.68\left(\mathrm{~m}, 4 \mathrm{H}, 2 \times \mathrm{SCH}_{2} \mathrm{CH}_{2}\right)$ ppm; ${ }^{13} \mathrm{C} \mathrm{NMR}\left(100 \mathrm{MHz}, \mathrm{CDCl}_{3}\right): \delta=53.9\left(-, 6 \times \mathrm{CCH}_{2} \mathrm{CH}\right), 44.7\left(\mathrm{C}_{\text {quart }}\right.$ $2 \times \mathrm{SC}), 30.8\left(-, 2 \times \mathrm{SCH}_{2} \mathrm{CH}_{2}\right), 29.7\left(-, 2 \times \mathrm{SCH}_{2} \mathrm{CH}_{2}\right), 28.8(+, 2 \times \mathrm{CH})$ ppm; IR (ATR): $\tilde{v}=2973(\mathrm{~m}), 2907(\mathrm{~m}), 2871(\mathrm{~m}), 1729(\mathrm{vw}), 1447(\mathrm{w})$, 
$1280(\mathrm{w}), 1205$ (s), $1139(\mathrm{~m}), 902(\mathrm{w}), 741(\mathrm{vw}), 442(\mathrm{vw}) \mathrm{cm}^{-1}$; MS (EI, $70 \mathrm{eV}): \mathrm{m} / z(\%)=254(3)[\mathrm{M}]^{+}, 155(23)\left[\mathrm{M}-\mathrm{C}_{5} \mathrm{H}_{7} \mathrm{~S}\right]^{+}, 147(62)\left[\mathrm{M}-\mathrm{C}_{5} \mathrm{H}_{7}-\right.$ $\left.\mathrm{C}_{3} \mathrm{H}_{5}+\mathrm{H}\right]^{+}, 120(10)\left[\mathrm{M}-\left(\mathrm{C}_{5} \mathrm{H}_{7}\right)_{2}\right]^{+}, 100(33)\left[\mathrm{C}_{5} \mathrm{H}_{7} \mathrm{~S}+\mathrm{H}\right]^{+}, 99(63)\left[\mathrm{C}_{5} \mathrm{H}_{7} \mathrm{~S}\right]^{+}$, 89 (56) [M- $\left.\mathrm{C}_{5} \mathrm{H}_{7}-\mathrm{C}_{5} \mathrm{H}_{7} \mathrm{~S}+\mathrm{H}\right]^{+}, 87(63)\left[\mathrm{C}_{4} \mathrm{H}_{7} \mathrm{~S}\right]^{+}, 85$ (95) $\left[\mathrm{M}-\mathrm{C}_{5} \mathrm{H}_{7} \mathrm{~S}_{-} \mathrm{C}_{4} \mathrm{H}_{8}-\right.$ $\left.\mathrm{CH}_{2}\right]^{+}, 67(85)\left[\mathrm{C}_{5} \mathrm{H}_{7}\right]^{+}, 55(100)\left[\mathrm{C}_{4} \mathrm{H}_{7}\right]^{+} ; \mathrm{HRMS}(\mathrm{El}, 70 \mathrm{eV}): \mathrm{C}_{14} \mathrm{H}_{22}{ }^{32} \mathrm{~S}_{2}[\mathrm{M}]^{+}$ 254.1157 ; found 254.1158 .

1,6-Bis(bicyclo[1.1.1]pent-1-ylthio)hexane (13c): 13c was synthesized from a solution of 1 (general procedure a) according to the general procedure c. The product $13 \mathrm{c}$ was obtained as a colourless liquid in $47 \%$ yield $(38.0 \mathrm{mg}, 135 \mu \mathrm{mol})$

${ }^{1} \mathrm{H} \mathrm{NMR}\left(400 \mathrm{MHz}, \mathrm{CDCl}_{3}\right): \delta=2.71(\mathrm{~s}, 2 \mathrm{H}, 2 \times \mathrm{CH}), 2.52(\mathrm{~m}, 4 \mathrm{H}$ $\left.2 \times \mathrm{SCH}_{2} \mathrm{CH}_{2} \mathrm{CH}_{2}\right), \quad 1.95\left(\mathrm{~s}, \quad 12 \mathrm{H}, \quad 6 \times \mathrm{CCH}_{2} \mathrm{CH}\right), \quad 1.59(\mathrm{~m}, \quad 4 \mathrm{H}$, $\left.2 \times \mathrm{SCH}_{2} \mathrm{CH}_{2} \mathrm{CH}_{2}\right), \quad 1.38\left(\mathrm{~m}, 4 \mathrm{H}, 2 \times \mathrm{SCH}_{2} \mathrm{CH}_{2} \mathrm{CH}_{2}\right) \quad \mathrm{ppm} ;{ }^{13} \mathrm{C} \mathrm{NMR}$ $\left(100 \mathrm{MHz}, \mathrm{CDCl}_{3}\right): \delta=53.8\left(-, 6 \times \mathrm{CCH}_{2} \mathrm{CH}\right), 44.7\left(\mathrm{C}_{\text {quart. }}, 2 \times \mathrm{SC}\right), 34.0$ $\left(-, 2 \times \mathrm{SCH}_{2} \mathrm{CH}_{2} \mathrm{CH}_{2}\right), 30.4\left(-, 2 \times \mathrm{SCH}_{2} \mathrm{CH}_{2} \mathrm{CH}_{2}\right), 28.8(+, 2 \times \mathrm{CH}), 28.1$ $\left(-, 2 \times \mathrm{SCH}_{2} \mathrm{CH}_{2} \mathrm{CH}_{2}\right)$ ppm; IR (ATR): $\tilde{v}=2974(\mathrm{w}), 2925(\mathrm{w}), 2872(\mathrm{w})$, $1727(\mathrm{vw}), \quad 1459(\mathrm{vw}), \quad 1270(\mathrm{vw}), \quad 1206(\mathrm{~m}), \quad 1141(\mathrm{w}), 903(\mathrm{vw})$, $736(\mathrm{vw}) \mathrm{cm}^{-1} ; \mathrm{MS}(\mathrm{El}, 70 \mathrm{eV}): \mathrm{m} / z(\%)=282(100)[\mathrm{M}]^{+}, 215(28)[\mathrm{M}-$ $\left.\mathrm{C}_{5} \mathrm{H}_{7}\right]^{+}, 148(11)\left[\mathrm{M}-\left(\mathrm{C}_{5} \mathrm{H}_{7} \mathrm{~S}\right)_{2}\right]^{+}, 100(46)\left[\mathrm{C}_{5} \mathrm{H}_{7} \mathrm{~S}+\mathrm{H}\right]^{+}, 99(96)\left[\mathrm{C}_{5} \mathrm{H}_{7} \mathrm{~S}\right]^{+}, 87$ (29) $\left[\mathrm{C}_{4} \mathrm{H}_{7} \mathrm{~S}\right]^{+}, 85(100)\left[\mathrm{M}-\mathrm{C}_{5} \mathrm{H}_{7} \mathrm{~S}-\mathrm{C}_{6} \mathrm{H}_{12}-\mathrm{CH}_{2}\right]^{+}, 67(81)\left[\mathrm{C}_{5} \mathrm{H}_{7}\right]^{+}$; HRMS $\left(\mathrm{El}, 70 \mathrm{eV}\right.$ ): calcd for $\mathrm{C}_{16} \mathrm{H}_{26}{ }^{32} \mathrm{~S}_{2}[\mathrm{M}]^{+}$282.1470; found 282.1472.

1-(Phenylsulfoxide)-bicyclo[1.1.1]pentane (14): In a $10 \mathrm{~mL}$ flask $100 \mathrm{mg}$ of 7a $(567 \mu \mathrm{mol}, 1.00$ equiv.) were dissolved in $1.0 \mathrm{~mL}$ dichloromethane. $87 \mathrm{mg} \mathrm{m}$-chloroperoxybenzoic acid (567 $\mu \mathrm{mol}, 1.00$ equiv.) were added in portions and the mixture was stirred for $5 \mathrm{~min}$ at room temperature. The precipitate was filtered off and the filtrate was washed with $\mathrm{Na}_{2} \mathrm{~S}_{2} \mathrm{O}_{3}$ solution and $1 \mathrm{M} \mathrm{NaOH}$-solution. The organic phase was dried over $\mathrm{Na}_{2} \mathrm{SO}_{4}$ and the solvent was removed under reduced pressure. The crude product was purified by column chromatography $\left(\mathrm{SiO}_{2}\right.$, cyclohexane/EtOAc, 5:1) to obtain the product $\mathbf{1 4}$ as a colourless oil in $71 \%$ yield $(77.0 \mathrm{mg}, 400 \mu \mathrm{mol})$.

$R_{\mathrm{ff}}\left(\mathrm{SiO}_{2}\right.$, cyclohexane/EtOAc, 5:1): 0.18; ${ }^{1} \mathrm{H} \mathrm{NMR}\left(400 \mathrm{MHz}, \mathrm{CDCl}_{3}\right)$ $\delta=7.53-7.47(\mathrm{~m}, 5 \mathrm{H}, \mathrm{Ar}-\mathrm{H}), 2.81(\mathrm{~s}, 1 \mathrm{H}, \mathrm{CH}), 1.88\left(\mathrm{~s}, 6 \mathrm{H}, 3 \times \mathrm{CH}_{2}\right) \mathrm{ppm}$; ${ }^{13} \mathrm{C}$ NMR $\left(100 \mathrm{MHz}, \mathrm{CDCl}_{3}\right) \delta=141.7$ (C quart, $\left.\mathrm{CAr}_{\mathrm{Ar}}\right), 130.9\left(+, \mathrm{CH}_{\mathrm{Ar}}\right), 129.0$ $\left(+, 2 \times \mathrm{CH}_{\mathrm{Ar}}\right), 124.3\left(+, 2 \times \mathrm{CH}_{\mathrm{Ar}}\right), 55.4\left(\mathrm{C}_{\text {quart, }} \mathrm{C}_{\mathrm{Ar}} \mathrm{SC}\right), 49.9\left(-, 3 \times \mathrm{CH}_{2}\right)$, $27.8(+, C H)$ ppm; IR (ATR): $\tilde{v}=3462(\mathrm{vw}), 2970(\mathrm{w}), 2917(\mathrm{w}), 2880(\mathrm{w})$, $1581(\mathrm{vw}), 1476(\mathrm{w}), 1443(\mathrm{w}), 1303(\mathrm{vw}), 1201(\mathrm{~m}), 1129(\mathrm{w}), 1084(\mathrm{~m})$, $1067(w), 1036(\mathrm{~m}), 997(\mathrm{~m}), 883(\mathrm{w}), 869(\mathrm{w}), 777(\mathrm{w}), 746(\mathrm{~m}), 692(\mathrm{~m})$, $555(\mathrm{~m}), 511(\mathrm{~m}), 484(\mathrm{~m}) \mathrm{cm}^{-1}$; MS (El, $\left.70 \mathrm{eV}\right): \mathrm{m} / z(\%)=192(2)[\mathrm{M}]^{+}$, $126(100)\left[\mathrm{M}-\mathrm{C}_{5} \mathrm{H}_{7}+\mathrm{H}\right]^{+}, 125(10)\left[\mathrm{M}-\mathrm{C}_{5} \mathrm{H}_{7}\right]^{+}, 78(41)\left[\mathrm{C}_{6} \mathrm{H}_{5}+\mathrm{H}\right]^{+}, 77(15)$ $\left[\mathrm{C}_{6} \mathrm{H}_{5}\right]^{+}, 67(61)\left[\mathrm{C}_{5} \mathrm{H}_{7}\right]^{+}$; HRMS (El, $70 \mathrm{eV}$ ): calcd for $\mathrm{C}_{11} \mathrm{H}_{12} \mathrm{O}^{32} \mathrm{~S}[\mathrm{M}]^{+}$ 192.0609; found 192.0610 .

1-(Phenylsulfonyl)-bicyclo[1.1.1]pentane (15): In a $10 \mathrm{~mL}$ flask $200 \mathrm{mg}$ of 7a $(1.13 \mathrm{mmol}, 1.00$ equiv.) were dissolved in $2.0 \mathrm{~mL}$ dichloromethane. $690 \mathrm{mg} \mathrm{m}$-chloroperoxybenzoic acid $(4.54 \mathrm{mmol}, 4.00$ equiv.) were added in portions and the mixture was stirred for $5 \mathrm{~min}$ at room temperature. The precipitate was filtered off and the filtrate was washed with $\mathrm{Na}_{2} \mathrm{~S}_{2} \mathrm{O}_{3}$ solution and $1 \mathrm{M} \mathrm{NaOH}$-solution. The organic phase was dried over $\mathrm{Na}_{2} \mathrm{SO}_{4}$ and the solvent was removed under reduced pressure to obtain the product 15 as a colourless oil in $69 \%$ yield $(162 \mathrm{mg}, 779 \mu \mathrm{mol})$.

${ }^{1} \mathrm{H} \mathrm{NMR}\left(400 \mathrm{MHz}, \mathrm{CDCl}_{3}\right): \delta=7.87-7.84(\mathrm{~m}, 2 \mathrm{H}, \mathrm{Ar}-\mathrm{H}), 7.67-7.63(\mathrm{~m}$, $1 \mathrm{H}, \mathrm{Ar}-\mathrm{H}), 7.58-7.54(\mathrm{~m}, 2 \mathrm{H}, \mathrm{Ar}-\mathrm{H}), 2.72(\mathrm{~s}, 1 \mathrm{H}, \mathrm{CH}), 2.08\left(\mathrm{~s}, 6 \mathrm{H}, 3 \times \mathrm{CH}_{2}\right)$ ppm; ${ }^{13} \mathrm{C} \mathrm{NMR}\left(100 \mathrm{MHz}, \mathrm{CDCl}_{3}\right) \delta=134.2$ (C quart, $\left.C_{\mathrm{Ar}}\right), 133.6(+$, $\left.2 \times \mathrm{CH}_{\mathrm{Ar}}\right), 128.9\left(+, 2 \times \mathrm{CH}_{\mathrm{Ar}}\right), 127.6\left(+, \mathrm{CH}_{\mathrm{Ar}}\right), 54.1\left(-, 3 \times \mathrm{CH}_{2}\right), 45.8$ (Cquart, $\left.\mathrm{C}_{\mathrm{Ar}} \mathrm{SC}\right), 28.8(+, \mathrm{CH}) \mathrm{ppm}$; IR (ATR): $\tilde{v}=2996(\mathrm{w}), 2918(\mathrm{w})$, $2884(\mathrm{w}), 1584(\mathrm{vw}), 1479(\mathrm{vw}), 1446(\mathrm{~m}), 1301(\mathrm{~s}), 1205(\mathrm{~m}), 1177(\mathrm{~m})$, $1130(\mathrm{~s}), 1078(\mathrm{~m}), 1022(\mathrm{w}), 998(\mathrm{w}), 940(\mathrm{w}), 898(\mathrm{w}), 876(\mathrm{~m}), 779(\mathrm{w})$ $759(\mathrm{~m}), 719(\mathrm{~m}), 689(\mathrm{~m}), 612(\mathrm{~s}), 564(\mathrm{~m}), 534(\mathrm{~m}) \mathrm{cm}^{-1} ; \mathrm{MS}$ (El, $\left.70 \mathrm{eV}\right):$ $\mathrm{m} / \mathrm{z}(\%)=209(1)[\mathrm{M}+\mathrm{H}]^{+}, 208(2)\left[\mathrm{M}^{+}, 191(2)[\mathrm{M}-\mathrm{OH}]^{+}, 143(27)[\mathrm{M}-\right.$ $\left.\mathrm{C}_{5} \mathrm{H}_{6}+\mathrm{H}\right]^{+}, 125(51)\left[\mathrm{M}-\mathrm{O}-\mathrm{C}_{5} \mathrm{H}_{7}\right]^{+}, 77(24)\left[\mathrm{C}_{6} \mathrm{H}_{5}\right]^{+}, 67(100)\left[\mathrm{C}_{5} \mathrm{H}_{7}\right]^{+} ; \mathrm{HRMS}$ $(\mathrm{El}, 70 \mathrm{eV})$ : calcd for $\mathrm{C}_{11} \mathrm{H}_{12}{ }^{32} \mathrm{~S}[\mathrm{M}]+1$ 176.0654; found 176.0655 .

Bicyclo[1.1.1]pent-1-yl(phenyl)selane (17): 17 was synthesized from a solution of 1 (general procedure b) according to the general procedure $\mathrm{c}$. Instead of a thiol benzeneselenol (16) was added as a 1 M solution in diethyl ether. The product $\mathbf{1 7}$ was obtained as a yellow liquid in quantitative yield $(133 \mathrm{mg}, 596 \mu \mathrm{mol}$ ).

${ }^{1} \mathrm{H}$ NMR $\left(400 \mathrm{MHz}, \mathrm{CDCl}_{3}\right): \delta=7.57-7.54(\mathrm{~m}, 2 \mathrm{H}, \mathrm{Ar}-\mathrm{H}), 7.30-7.25(\mathrm{~m}$, $3 \mathrm{H}, \mathrm{Ar}-\mathrm{H}), 2.96(\mathrm{~s}, 1 \mathrm{H}, \mathrm{CH}), 2.00\left(\mathrm{~s}, 6 \mathrm{H}, 3 \times \mathrm{CH}_{2}\right) \mathrm{ppm} ;{ }^{13} \mathrm{C} \mathrm{NMR}(100 \mathrm{MHz}$, $\left.\mathrm{CDCl}_{3}\right): \delta=135.4\left(+, 2 \times \mathrm{CH}_{\mathrm{Ar}}\right), 131.7$ (C quart.,$\left.C_{\mathrm{Ar}}\right), 128.9\left(+, 2 \times \mathrm{CH}_{\mathrm{Ar}}\right)$, $127.6\left(+, \mathrm{CH}_{\mathrm{Ar}}\right), 55.4\left(-, 3 \times \mathrm{CH}_{2}\right), 38.9$ (Cquart. $\left.\mathrm{C}_{\mathrm{Ar}} \mathrm{SeC}\right), 31.0(+, \mathrm{CH}) \mathrm{ppm}$; IR (ATR): $\tilde{v}=3056(\mathrm{vw}), 2962(\mathrm{w}), 2909(\mathrm{w}), 2874(\mathrm{w}), \quad 1577(\mathrm{w})$ $1475(\mathrm{~m}), 1436(\mathrm{w}), 1299(\mathrm{w}), 1204(\mathrm{~m}), 1117(\mathrm{~m}), 1072(\mathrm{w}), 1021(\mathrm{w})$, $999(\mathrm{w}), 883(\mathrm{~m}), 735(\mathrm{~m}), 690(\mathrm{~m}), 671(\mathrm{w}), 471(\mathrm{w}) \mathrm{cm}^{-1}$; MS (El, $\left.70 \mathrm{eV}\right)$ : $\mathrm{m} / \mathrm{z}(\%)=224 / 222 / 220(18 / 19 / 5)[\mathrm{M}]^{+}, 158(21)\left[\mathrm{M}-\mathrm{C}_{5} \mathrm{H}_{7}+\mathrm{H}\right]^{+}, 157(20)[\mathrm{M}-$ $\left.\mathrm{C}_{5} \mathrm{H}_{7}\right]^{+}, 78(33)\left[\mathrm{C}_{6} \mathrm{H}_{5}+\mathrm{H}\right]^{+}, 77(41)\left[\mathrm{C}_{6} \mathrm{H}_{5}\right]^{+}, 67(100)\left[\mathrm{C}_{5} \mathrm{H}_{7}\right]^{+} ; \mathrm{HRMS}(\mathrm{EI}$, $70 \mathrm{eV}$ ): calcd for $\mathrm{C}_{11} \mathrm{H}_{12}{ }^{80} \mathrm{Se}[\mathrm{M}]^{+} 224.0104$; found 224.0104 .

\section{Acknowledgements}

We greatly acknowledge the contribution of the precursor 1,1-dibromo-2,2-bis(chloromethyl)cyclopropane (3) by abcr $\mathrm{GmbH}$. R.M.B. acknowledges the SFB 1176 funded by the German Research Council (DFG) in the context of project B3 for funding.

Keywords: [1.1.1]propellane $\cdot$ bicyclo[1.1.1]pentane $\bullet$ thiols $•$ hydrogen sulfide $\cdot$ amino acid

[1] a) A. M. Dilmaç, E. Spuling, A. de Meijere, S. Bräse, Angew. Chem. 2017, 129, 5778-5813; b) A. M. Dilmaç, E. Spuling, A. de Meijere, S. Bräse Angew. Chem. Int. Ed. 2017, 56, 5684-5718.

[2] J. Altman, E. Babad, J. Itzchaki, D. Ginsburg, Tetrahedron 1966, 22 279-304.

[3] a) K. B. Wiberg, J. Am. Chem. Soc. 1983, 105, 1227-1233; b) K. B. Wiberg, W. P. Dailey, F. H. Walker, S. T. Waddell, L. S. Crocker, M. Newton, J. Am. Chem. Soc. 1985, 107, 7247-7257; c) K. B. Wiberg Chem. Rev. 1989, 89, 975-983.

[4] a) K. B. Wiberg, F. H. Walker, J. Am. Chem. Soc. 1982, 104, 5239-5240; b) E. Honegger, H. Huber, E. Heilbronner, W. P. Dailey, K. B. Wiberg, J. Am. Chem. Soc. 1985, 107, 7172-7174; c) A. M. Orendt, J. C. Facelli, D. M. Grant, J. Michl, F. H. Walker, W. P. Dailey, S. T. Waddell, K. B. Wiberg, M. Schindler, W. Kutzelnigg, Theor. Chim. Acta 1985, 68, 421-430; d) L. Hedberg, K. Hedberg, J. Am. Chem. Soc. 1985, 107, 7257-7260.

[5] K. B. Wiberg, S. T. Waddell, J. Am. Chem. Soc. 1990, 112, 2194-2216.

[6] K. Semmler, G. Szeimies, J. Belzner, J. Am. Chem. Soc. 1985, 107, 6410-6411.

[7] a) P. Kaszynski, J. Michl, J. Am. Chem. Soc. 1988, 110, 5225-5226; b) P. Kaszynski, A. C. Friedli, J. Michl, J. Am. Chem. Soc. 1992, 114, 601620.

[8] P. Kaszynski, A. C. Friedli, J. Micul, Mol. Cryst. Liq. Cryst. Lett. 1988, 6 , 27-33.

[9] P. F. Schwab, B. C. Noll, J. Michl, J. Org. Chem. 2002, 67, 5476-5485

[10] a) A. F. Stepan, C. Subramanyam, I. V. Efremov, J. K. Dutra, T. J. O'Sullivan, K. J. DiRico, W. S. McDonald, A. Won, P. H. Dorff, C. E. Nolan, S. L. Becker, L. R. Pustilnik, D. R. Riddell, G. W. Kauffman, B. L. Kormos, L. Zhang, Y. Lu, S. H. Capetta, M. E. Green, K. Karki, E. Sibley, K. P. 
Atchison, A. J. Hallgren, C. E. Oborski, A. E. Robshaw, B. Sneed, C. J. O'Donnell, J. Med. Chem. 2012, 55, 3414-3424; b) K. C. Nicolaou, D. Vourloumis, S. Totokotsopoulos, A. Papakyriakou, H. Karsunky, H. Fernando, J. Gavrilyuk, D. Webb, A. F. Stepan, ChemMedChem 2016 11, 31-37; c) N. D. Measom, K. D. Down, D. J. Hirst, C. Jamieson, E. S. Manas, V. K. Patel, D. O. Somers, ACS Med. Chem. Lett. 2017, 8, 4348; d) Y. P. Auberson, C. Brocklehurst, M. Furegati, T. C. Fessard, G. Koch, A. Decker, L. La Vecchia, E. Briard, ChemMedChem 2017, 12, 590-598.

[11] I. S. Makarov, C. E. Brocklehurst, K. Karaghiosoff, G. Koch, P. Knochel, Angew. Chem. Int. Ed. 2017, 56, 12774-12777.

[12] K. D. Bunker, N. W. Sach, Q. Huang, P. F. Richardson, Org. Lett. 2011 13, 4746-4748.

[13] R. Gianatassio, J. M. Lopchuk, J. Wang, C. M. Pan, L. R. Malins, L. Prieto, T. A. Brandt, M. R. Collins, G. M. Gallego, N. W. Sach, J. E. Spangler, H. Zhu, J. Zhu, P. S. Baran, Science 2016, 351, 241-246.

[14] J. A. Milligan, P. Wipf, Nat. Chem. 2016, 8, 296-297.

[15] K. R. Mondanaro, W. P. Dailey, Org. Synth. 1998, 75, 98-105.

[16] a) K. B. Wiberg, S. T. Waddell, K. Laidig, Tetrahedron Lett. 1986, 27, 1553-1556; b) K. B. Wiberg, S. T. Waddell, Tetrahedron Lett. 1988, 29 289-292; c) U. Bunz, K. Polborn, H. U. Wagner, G. Szeimies, Chem. Ber. 1988, 121, 1785-1790; d) P. Kaszynski, A. C. Friedli, J. Michl, Mol. Cryst Liq. Cryst. Lett. 1988, 6, 27-33; e) A. C. Friedli, P. Kaszynski, J. Michl Tetrahedron Lett. 1989, 30, 455-458; f) V. Vasin, I. Y. Bolusheva, E. P. Sanaeva, L. Surmina, N. Sadovaya, A. Koz'min, N. Zefirov, Dokl. Akad. Nauk SSSR 1989, 305, 621-624; g) J. Michl, P. Kaszynski, A. C. Friedli, University of Texas System, USA, 1989, p. 77 pp; h) A. Friedli, V. Lynch,
P. Kaszynski, J. Michl, Acta Crystallogr., Sect. A 1990, 46, 377-389; i) P Kaszynski, N. D. McMurdie, J. Michl, J. Org. Chem. 1991, 56, 307-316; j) D. S. Toops, M. R. Barbachyn, J. Org. Chem. 1993, 58, 6505-6508; k) E. W. Della, I. J. Lochert, N. M. Peruchena, G. A. Aucar, R. H. Contreras, J. Phys. Org. Chem. 1996, 9, 168-178; I) P. McGarry, J. Scaiano, Can. J. Chem. 1998, 76, 1474-1489; m) M. T. Hossain, J. W. Timberlake, J. Org. Chem. 2001, 66, 6282-6285; n) K. D. Bunker, Kalyra Pharmaceuticals, Inc., USA, 2015, p. 40pp; o) K. D. Bunker, Kalyra Pharmaceuticals, Inc., USA, 2015, p. 37pp; p) K. D. Bunker, C. Guo, M. C. Grier, C. D. Hopkins, J. R. Pinchman, D. H. Slee, Q. Huang, M. Kahraman, Kalyra Pharmaceuticals, Inc., USA, 2016, p. 53pp.

[17] J. M. Lopchuk, K. Fjelbye, Y. Kawamata, L. R. Malins, C.-M. Pan, R. Gianatassio, J. Wang, L. Prieto, J. Bradow, T. A. Brandt, M. R. Collins, J. Elleraas, J. Ewanicki, W. Farrell, O. O. Fadeyi, G. M. Gallego, J. J. Mousseau, R. Oliver, N. W. Sach, J. K. Smith, J. E. Spangler, H. Zhu, J. Zhu, P. S. Baran, J. Am. Chem. Soc. 2017, 139, 3209-3226.

[18] E. W. Della, P. E. Pigou, C. H. Schiesser, D. K. Taylor, J. Org. Chem. 1991, 56, 4659-4664.

[19] a) P. F. McGarry, L. J. Johnston, J. C. Scaiano, J. Org. Chem. 1989, 54, 6133-6135; b) P. F. McGarry, J. C. Scaiano, Can. J. Chem. 1998, 76, 1474-1489.

[20] K. B. Wiberg, D. S. Connor, J. Am. Chem. Soc. 1966, 88, 4437-4441.

[21] a) A. C. Friedli, V. M. Lynch, P. Kaszynski, J. Michl, Acta Crystallogr., Sect. A 1990, 46, 377-389; b) S. Mazières, M. K. Raymond, G. Raabe, A. Prodi, J. Michl, J. Am. Chem. Soc. 1997, 119, 6682-6683.

[22] H. Okamura, C. Bolm, Chem. Lett. 2004, 33, 482-487. 
Entry for the Table of Contents (Please choose one layout)

Layout 1:

\section{FULL PAPER}

\section{A versatile tool for new building} blocks. Thiols can open

[1.1.1]propellane in simple, clean and fast reactions with good functional group tolerance. Even hydrogen sulfide, amino acids and selenols can be used in this radical reaction. The products can be further modified to tune the polarity. This reaction can potentially be applied in material modifications, bioconjugations or in the synthesis of new medicinal chemistry building blocks.

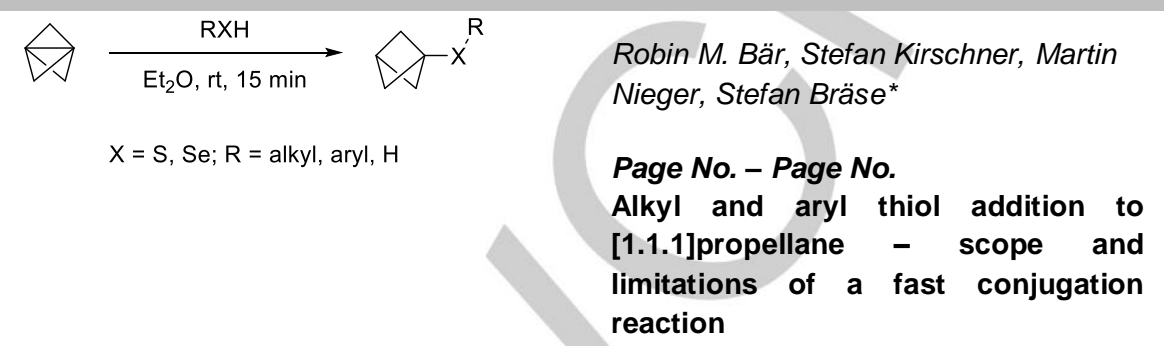

Robin M. Bär, Stefan Kirschner, Martin

Page No. - Page No.

[1.1.1]propellane - scope and reaction 